\title{
STAAT UND KLASSE IN PERIPHER-KAPITALISTISCHEN GESELLSCHAFTSFORMATIONEN: DIE ENTWICKLUNG DES ABHÄNGIGEN STAATSKAPITALISMUS IN AFRIKA
}

\author{
Von Rainer TetzlafF
}

\section{Die Funktions- und Klassenbestimmung des peripheren Staates als Forschungsproblem*}

Für die aktuelle sozialwissenschaftliche Diskussion über Entwicklungsstrategien und Entwicklungsperspektiven in der sog. Dritten Welt sind heute zwei Fragen von konstitutiver Bedeutung: erstens die Frage nach der realen und potentiellen Rolle „des Staates" im Prozeß einer gesunden gesamtgesellschaftlichen Entwicklung und zweitens die Frage nach den Veränderungschancen der gegenwärtig entwicklungshemmenden Klassenstrukturen in weltmarktabhängigen Ländern mit peripher-kapitalistischen Gesellschaftsformationen. Im folgenden wird von der Prämisse ausgegangen, daß beide Fragen nur gemeinsam in wechselseitiger Beziehung zueinander analysiert werden können und darüber hinaus nur im jeweils spezifischen historischen Milieu der internationalen Entwicklung. Damit distanzieren sich die folgenden Überlegungen von einem unhistorischen Erkenntnisinteresse, das sich verkürzt von der Frage leiten läßt: „Welchen Handlungsspielraum nach innen und außen besitzt der Staatsapparat in Entwicklungsländern?" (dazu A. Schmidt 1976, 16). Ergiebiger erscheint uns die Problemperzeption von Mamalakis und Wioczek: Ist die regierende Klasse „eine technokratische Entität mit einem Eigenleben; ist sie eine Widerspiegelung der Kräftebalance zwischen verschiedenen soziopolitischen Gruppen; oder ist sie bloß ein Instrument in den Händen einiger dominierender Machtgruppen, die sich ihrer auf verschiedene Art und Weise bedienen?" (nach H. Grewe 1974, 35).

$\mathrm{Da}$ es sich hier um Gesellschaftsformationen handelt, die bei aller strukturellen Heterogenität der Produktionsverhältnisse doch von der kapitalistischen Produktionsweise dominiert werden und deren gemeinsames geschichtliches Erbe die abhängige Integration in einen imperialistischen Weltmarkt mit vertikaler internationaler Arbeitsteilung ist (vgl. z. B. Senghaas 1974), ist u. E. der peripher-kapitalistische Staat ebenso wie der der kapitalistischen Metropolen primär - d. h. aber keineswegs ausschließlich - als Instrument einer Klassenherrschaft $\mathrm{zu}$ analysieren und nicht etwa hauptsächlich als rational verwendbares Interventionsinstrument von politisch neutralen Technokraten. Für die Peripherien ist hierbei die Frage von zentraler Bedeutung: welche Abhängigkeitsbeziehungen entwickeln sich zwischen der regierenden lokalen Staatsbürokratie und den herrschenden Klassen und Klassenfraktionen? Gibt es eine deutlich erkennbare "Staatsklasse“, und wie löst sie im konkreten Fall den objektiven Zielkonflikt zwischen der Stabilisierung des politischen und sozialen Herrschaftssystems und seiner politischen Legitimierung, die kurzfristig durch angemessene Ressourcenverteilung, langfristig aber nur durch sozio-ökonomische „Effizienz“, d. h. Veränderung des Produktionssystems, zu erreichen ist? Dabei ist zu

\footnotetext{
* Harald Sellin, Barbara Töpper, Renate Nestvogel und den Kollegen des Arbeitskreises „Entwicklungstheorie und Entwicklungspolitik" der Deutschen Vereinigung für Politische Wissenschaft bin ich für Anregungen und kritische Kommentare zu Dank verpflichtet.
} 
problematisieren, ob "der Staatsapparat“ in Peripherie-Ländern tatsächlich — bzw. unter welchen sozialen Bedingungen - a priori als der eigentliche und zentrale Agent für Entwicklung apostrophiert werden kann. Nur allzu häufig bleibt auch in Überlegungen über „alternative" Entwicklungsstrategien die historisch-dialektische Vermittlung von sich entwickelnden Klassenwidersprüchen mit den (modellhaft postulierten, aktiven oder passiven) „Verhalten“ von Staatsapparaten auf der Strecke. Unter der Hand wird der Staat zum „Deus ex machina“ hypostasiert, der, wenn er nur wollte, auch tun könnte, was er sollte!

Demnach müßte die praktisch relevante Frage bezüglich der Möglichkeiten und Grenzen von Staatstätigkeiten angesichts der historisch zunächst vorgegebenen internen wie externen Produktionsverhältnisse (bei einem je spezifischen Typ von Entwicklungsland) lauten: Welches Klassenbündnis kann mittels der Staatsmacht „Entwicklung“ initiieren? Dabei wird „Entwicklung“ im Sinne eines normativen Ziels definiert als ein umfassender Veränderungsprozeß, der den unteren Zweidrittel der Bevölkerung eines Entwicklungslandes (EL) eine spürbare materielle und geistige Existenzverbesserung ermöglicht. Dies ist ohne die schnelle Steigerung der gesamtgesellschaftlichen Produktivität der Arbeit undenkbar. Aus dieser Fragestellung ergibt sich die analyseleitende Erkenntnis, daß nicht der periphere Staat als außerökonomische Organisationspotenz oder als systemgarantierendes Interventionsinstrument, d. h. als Leistungsstaat, erstes Objekt der Analyse von Entwicklungsmöglichkeiten in der Dritten Welt sein kann, sondern daß alle Staatstätigkeit als eine abgeleitete Größe zu begreifen ist, der per se kein Wert als Entwicklungsinstrument zukommt. Sie ist hier primär als Ausdruck bestimmter dominanter Interessen und Zwangslagen einer (als afrikanische „Staatsbourgeoisie“ näher zu konkretisierenden) „Klasse“ abzuleiten und zu erklären. Die Staatsanalyse erhält erst durch die Klassenanalyse ihren Sinn. Ferner ist Ocampo und Johnson zuzustimmen, daß „jede Theorie über Politik und Entwicklungsprozeß die Art und Weise berücksichtigen muß, in der Macht, die sich in der Kontrolle über die Volkswirtschaft und den Machtressourcen sozialer Klassen abzeichnet, sich im Staat widerspiegelt. Das Hauptproblem ist dabei die Identifizierung der verschiedenen Mechanismen, anhand derer die ökonomische Macht dominierender Gesellschaftsklassen übertragen wird in institutionalisierte politische Macht" (1972, 408).

In der gegenwärtigen Staatsdiskussion ist es weitverbreitet, die Rolle und Funktion des peripher-kapitalistischen Staatsapparates im Entwicklungsprozeß mit den (oberflächlichen) Kategorien von "Stärke" und "Schwäche" gegenüber einheimischen und vor allem ausländischen Wirtschafts- und Gesellschaftsinteressen zu bestimmen. Handelt es sich bei den einen um sozial weitgehend unabhängige, politisch „relativ autonome“ Staaten (H. Alawi, R. Sonntag etc) mit potentiell großer politischer „bargaining power" gegenüber multinationalen Konzernen und den Metropolen (H. Elsenhans), so schätzen andere Wissenschaftler die Entwicklungsländer als per se "schwache Staaten“ ein (G. Myrdal), die institutionell, personell und finanziell als fragil und fremdgelenkt erscheinen. Auch Barnet und Müller betonen die institutionellen Schwächen des Staates in den EL, wenn sie pauschal konstatieren: „Die schwache Verhandlungsbasis der unterentwickelten Länder beruht also auf drei fundamentalen institutionellen Schwächen. Die erste sahen wir in den antiquierten Regierungsstrukturen. Die Gesetze taugen nicht dazu, Steuern einzutreiben, ausländische Unternehmen zu kontrollieren oder den Abfluß des Finanzkapitals zu verhindern ... Eine zweite Quelle der institutionellen Schwäche unterentwickelter Länder ist das 
Fehlen einer starken Gewerkschaftsbewegung ... Eine dritte Quelle der institutionellen Schwäche ist die mangelnde Konkurrenz einheimischer Geschäftsleute. Auch darum kann der multinationale Konzern in unterentwickelten Ländern sowiel Macht ausüben ...

Die Weltkonzerne (können) das einheimische Finanzkapital und den eigenen technologischen Vorteil benutzen, um die einheimische Industrie zu absorbieren. Das ist ein Prozeß, den manche einheimischen Geschäftsleute vielleicht beklagen, aber bis heute noch nicht aufhalten können" (Barnet/Müller 1975, 120-123). Immerhin wird hier bereits die institutionelle Schwäche des Staates auch im Zusammenhang mit der unterentwickelten Gesellschaftsstruktur gesehen.

Der bisher anspruchsvollste (deutschsprachige) Beitrag zu dieser Problematik ist wohl - neben dem Diskussionspapier von T. Evers - der Aufsatz von W. Hein und G. Simonis über „Entwicklungspolitik, Staatsfunktionen und Klassenauseinandersetzungen im peripheren Kapitalismus" (Brasilien und Chile). Darin verfolgen die Autoren das Ziel, „logisch-rekonstruktive und historisch-konkrete Analysen miteinander zu verbinden, um praktisch relevante Aussagen treffen zu können" $(1976,217)$. Für sie ergeben sich „aus der Bestimmung des peripheren Kapitalismus als besonderer kapitalistischer Gesellschaftsform“ „drei wichtige, in der gegenwärtigen Debatte um den peripheren Staat [genannt werden: Alavi, Sonntag, Hein/Stenzel, Gerstenberger, Saul] nahezu unberücksichtigte [?] theoretisch-methodische Konsequenzen“: Eine Theorie des peripheren Staates setze erstens „die Theorie des kapitalistischen Staates im allgemeinen“ voraus; zweitens seien „die allgemeinen Struktur- und Funktionsbestimmungen des kapitalistischen Staates zu konkretisieren“, um einen „Begriff des kapitalistischen Staates der Peripherie“ zu gewinnen, der sich von der „theoretischen Bestimmung des Staates in entwickelten zentralen kapitalistischen Gesellschaften unterscheidet“; und schließlich kann die geforderte „Konkretisierung der abstrakten Theorie“ nur durch "die Berücksichtigung der besonderen historischen Erscheinung des Vergesellschaftungsprozesses, von gesellschaftlichen Krisen und vor allem der Klassenkämpfe erfolgen" (218/9). So nützlich der Rekurs auf allgemeine Problemfelder, "die besondere Staatsfunktionen" hervorbringen (Rechtsstaatlichkeit, allgemeine Produktionsbedingungen etc.), sein mag, die angekündigte historische Konkretion eines besonderen Begriffs von „kapitalistischem Staat der Peripherie“ gelingt auf diese Weise noch nicht. Abstrakt-logisch aus der "strukturellen Heterogenität“ der peripheren Gesellschaft deduzierte Thesen über den peripheren Staat tragen oft entweder rein postulativen Charakter oder bedeuten ans Tautologische grenzende Auffächerungen des Begriffes, z. B.: „Staatliche Industrialisierungspolitik ist im peripheren Kapitalismus unter den gegenwärtigen internen und Weltmarktbedingungen funktionaler Imperativ" (224/225). Ebenso wenig kann die Feststellung befriedigen, daß die „strukturellen Ursachen“, die „die Erscheinungsformen der ,Schwäche des peripheren Staates erklären können“ („administrative Irrationalität, politische Instabilität und Abhängigkeit"), die folgenden drei sein sollen:

(a) seine „geringe Legitimationsbasis“ („gering“ im Vergleich wozu?),

(b) seine "fraktionierte und instabile Klassenstruktur" und

(c) „die unmittelbare, kontinuierliche und widersprüchliche Intervention in den Reproduktionsprozeß, die eine Politisierung der Ökonomie auslöst und Privatisierungstendenzen des Staatsapparates begünstigt" (226/7; wo liegt der Unterschied zum kapitalistischen Staat und ist die „unmittelbare . . . Intervention “ tatsächlich eine „strukturelle Ursache“ für die Schwäche des peripheren Staates?). Allerdings ist 
die Hypothese, daß „der Staat der Peripherie . . . unmittelbar als Agent der herrschenden Klasse" erscheint (227), einer Überprüfung wert, lenkt sie doch die Aufmerksamkeit auf wichtige Fragen: inwieweit sind lokale Staatsfunktionäre und ökonomisch herrschende Klasse in EL personell und interessenmäßig identisch bzw. verquickt; übt der periphere Staat direkt ökonomische Funktionen (als Unternehmer) aus, die über die politische und rechtliche und ideologische Organisation des Gesamtsystems hinausgehen; ist die lokale "Staatsklasse“ als der direkte (oder nur indirekte, vermittelte) Feind der unterdrückten Klassen anzusehen?

Die Durchsicht der relevanten Literatur ergibt hierbei folgenden Befund: einerseits wird die umfassende „Schwäche des Staates“ in den Peripherien konstatiert, andererseits wird gerade vom relativ autonomen Staatsapparat als die zentrale Macht- und planungsfähige Entscheidungsinstanz die politisch forcierte Entwicklung der Produktivkräfte erwartet (R. Green/A. Seidman, E. Okwuosa) - ohne daß staatliches Handeln mit der je spezifischen Klassenstruktur vermittelt erscheint. Zur Auflösung dieses scheinbaren Paradoxons kann folgende empirisch verifizierbare Beobachtung beitragen: Nirgendwo tritt der periphere Staat in der Form des bürgerlich demokratischen und parlamentarischen Rechtsstaates in Erscheinung, sondern üblicherweise in der fragilen Hülle des zivilen oder militärischen Einpartei- oder Keinparteiregimes. Diese instabile institutionelle Form des peripheren Staates wie auch sein oft gnadenlos eingesetztes starkes Repressionspotential nach innen ergeben sich offensichtlich aus dem historischen Charakter der krisenhaften Produktionsverhältnisse, d. h. ihrer strukturellen Heterogenität: das Kapitalverhältnis hat sich noch nicht voll durchgesetzt und damit auch nicht die Verkehrsformen freier konkurrierender Warenbesitzer (vgl. T. Evers).

Daraus ergibt sich immerhin eine weitere, wenn auch noch grobe politische Ortsbestimmung der "Staatsklasse“ in der peripher-kapitalistischen Entwicklungsgesellschaft zwischen den zentralen internationalen Akteuren und den beherrschten einheimischen Gruppen: Der innere politische Handlungsspielraum der "Staatsklasse“ — deren Zusammensetzung es jeweils historisch-konkret zu bestimmen gilt - gegenüber der Mehrheit der einheimischen Bevölkerung ist relativ groß - relativ zu dem, was heute in den kapitalistischen Metropolen an Systemsicherung möglich und notwendig ist und meßbar am hohen Grad der Repressionsgewalt wie aber auch am Erfolg, sich gesellschaftlichen Surplus anzueignen. Das nach außen gerichtete Aktionspotential des peripheren Staates - d. h. in Richtung auf Reduktion von Weltmarktabhängigkeit und bezüglich eines rigiden Wirtschaftsnationalismus - ist absolut gering, weil bei jedem (nicht-revolutionären) Versuch, die Systemgrenzen zu seinen Gunsten zu verändern, der periphere Staat sofort und massiv seine "Souveränitätsgrenzen“ zu spüren bekommt. Die gescheiterten ökonomischen Emanzipationsversuche von drei afrikanischen Staaten in den 60er Jahren (vgl. Grohs/Tibi), die im Namen des afrikanischen „Sozialismus" unternommen wurden, - Ägypten, Guinea, Ghana —, haben die engen Handlungsschranken staatskapitalistischer Bürokratien deutlich demonstriert (Lehrstücke ähnlicher Art liefern Chile, Portugal, Peru). Das politische Drama hat hierbei folgende Logik: in dem Augenblick, in dem der periphere Staat die Spielregeln seiner Integration in das hierarchische Weltmarktsystem zu seinen Gunsten zu verändern droht, treten jene international operierenden Akteure aus der Reserve, die in allen peripher-kapitalistischen Ländern mehr oder weniger sichtbar wichtige staatliche Ersatz- und Ergänzungsfunktionen ausüben (womit nationale Souveränität zum bloßen Schein zu verkümmern tendiert): 
- der Internationale Währungsfonds und die Weltbank,

- die Staatsadministrationen in den Metropolen,

- das internationale private Busineß: multinationale Konzerne, multinationale Banken und technische Servicegesellschaften.

Ihre Gesamtheit bildet das, was im folgenden „metropolitane Bourgeoisie“ genannt werden soll. Während die einen die internationale Kreditwürdigkeit und Zahlungsfähigkeit des EL bestimmen und damit erheblichen Einfluß auf Entscheidungen im monetären und finanzpolitischen Bereich nehmen, verfügen die anderen über die Modalitäten seiner Reproduktion: Nahrungsmittellieferungen können gewährt oder verzögert (PL - 480 - Praktiken der USA), technisches Know-how kann geliefert oder vorenthalten, Handelsbeziehungen können liberalisiert (z. B. wie im LoméAbkommen der Europäischen Gemeinschaft mit $46 \mathrm{EL}$ ) oder künstlich erschwert werden (Exportrestriktionsklauseln in Joint-Venture-Verträgen) etc.

Daraus ist die Hypothese zu gewinnen: Soweit der lokale Staatsapparat im EL überhaupt Staatsfunktionen zur Regulierung wirtschaftlicher Prozesse auszuüben vermag, handelt er als Teil eines (fiktiven) strukturell heterogenen Staates, dessen politisches Entscheidungszentrum nicht mit nationalstaatlichen Kategorien zu erfassen ist, sondern in der Suprastruktur der internationalen Herrschaftsbeziehungen aufgespürt werden müßte. Angesichts der (zunächst hypothetischen) Entnationalisierung und geographischen Dezentralisierung von Staatsfunktionen in den EL (als Folge der Internationalisierung der Produktion und der dementsprechenden Ausdifferenzierung von Staatsfunktionen) ist problematisch, ob. sich nationale Handlungsspielräume und Systemgrenzen der "Staatsklassen“ in peripher-kapitalistischen Gesellschaften überhaupt einigermaßen genau bestimmen lassen. Wenn offenbar zentrale Bereiche staatlicher Interventionspolitik quasi ausgelagert sind - eben auf jene international operierenden Institutionen mit staatlichen Ersatz- und Ergänzungsfunktionen -, dann müßte Staatsanalyse in EL primär die Analyse der institutionalisierten Interessensynthese aus lokalen und internationalen Bourgeoisiefraktionen b.edeuten.

Im Lichte dieser Überlegungen ist auch die These von der „territorialen Deckungsungleichheit" (R. Murray) zwischen (national beschränkter) politischer Machtsphäre und der (internationalen) ökonomischen Sphäre - bezüglich der Dritte-Welt-Staaten - noch einmal zu überdenken. Auch T. Evers spricht in diesem Zusammenhang von „hierarchischer Dialektik“: „Die Inkongruenz von Basis und Überbau führt notwendig zu einer nichtreziproken Dialektik zwischen beiden: die ökonomische Basis . . . ist eine andere als diejenige, auf die der Staat zurückzuwirken vermag: Erstere ist eine Totalität, letztere ein Teil daraus" $(1976,73)$. Läßt sich dagegen die Hypothese von einer zunehmenden Internationalisierung der Staatsfunktionen in EL empirisch nachweisen und theoretisch absichern, so müßte eher von einer neuen Kongruenz gesprochen werden, die sich zwischen Basis und Überbau, konkret, zwischen internationalisierter OKonomie und internationalisierter Herrschaftsstruktur als globales Puzzle von nationalen und transnationalen Dominanz- und Dependenzbeziehungen tendenziell und prozeßhaft herstellt. Die sich daraus ergebenden Konsequenzen für die Analyse der staatlich induzierten Entwicklungsdynamik in Peripheriegesellschaften liegen auf der Hand: sie entzieht sich mit zunehmender abhängiger Entwicklung (Industrialisierung) immer mehr eines rein länderbezogenen (regionalspezifischen) $\mathrm{Zu}$ griffs und muß sich der Frage stellen, ob nicht die spezifische Aufgabe des peripheren Staates darin besteht, eine grenzüberschreitende Schanier- und Vermittlungsfunktion in Abhängigkeit von den dominanten Zentren auszuüben, d. h. die lokalen Produk- 
tionsverhältnisse an die Bedürfnisse der Metropolen anzupassen, was eine Nutzung freiwerdender Produktionsspielräume (aufgrund des Strukturwandels in den Metropolen), allerdings in Konkurrenz mit anderen peripheren Staaten mit alternativen Produktionszonen, nicht ausschlösse. Damit würde es auch problematisch werden, (mit Blanke, Jürgen/Kastendiek) zwischen Tätigkeitsgrenzen und Systemgrenzen von staatlichen Akteuren kategorial streng unterscheiden zu wollen; denn jede Tätigkeit des abhängigen Staates ist durch internationale Systemstrukturen (u. a. Weltmarktentwicklung) nicht nur beeinflußt, sondern sogar weitgehend vermittelt und mitbestimmt. Dabei scheint es allerdings eine Einschränkung zu geben: bewaffnete und klassenunspezifische Staatsadministrationen in Peripherieländern (vom Typ Uganda unter Präsident Amin) können schwerlich (von innen oder von außen) davon abgehalten werden, gegen die (kapitalistische) Logik des Gesamtsystems - von dem sie objektiv ein integraler Teil sind $-\mathrm{zu}$ handeln. Allerdings bedeutet ein solcher Selbstaustritt eines Landes aus der Hierarchie der internationalen Arbeitsteilung soweit überhaupt voll realisierbar - noch nicht Entwicklung, sondern lediglich Stillstand der Dynamik der bisherigen Unterentwicklungsmechanismen, was freilich oft mit neuen Abhängigkeiten erkauft wird. Politische Willkür ist nicht gleichbedeutend mit politischer Autonomie!

Immerhin deutet die Vielfalt politischer Herrschafts- und Regimeformen in Afrika (formale Mehrparteiensysteme, offen repressive Einparteidiktaturen, populistische oder charismatisch geführte Militärregimes, quasi-feudale Monarchien, demokratische Volksrepubliken etc.) darauf hin, daß bei relativer Gleichartigkeit der kolonialen Vergangenheit für die jeweilige Staatsklasse keine starr vorgegebenen, unverrückbaren Tätigkeitsgrenzen existieren. Im Gegenteil, innerhalb des Rahmens der internationalen Systemstruktur scheint - abhängig vom Typ des Entwicklungslandes (s. u.) - ein unterschiedlich großer staatlicher Manövrierraum für mehr oder weniger massive Eingriffe in die Produktions- und Zirkulationssphäre, in Eigentumsund Rechtsverhältnisse der Gesellschaft, vorhanden zu sein - Interventionen, die letztlich den oben genannten politischen Formationen ihren konkreten gesellschaftlichen Gehalt verleihen. Andererseits signalisiert die relative Gleichförmigkeit und Dauerhaftigkeit der grundlegenden Entwicklungsprobleme und -krisen in fast allen Staaten Afrikas (Agrarstrukturkrise, Marginalisierung von Arbeitskraft, Außenverschuldung, Militarisierung etc.), daß die Wirksamkeit staatlicher Interventionen in Wirtschaft und Gesellschaft (in Hinblick auf entwicklungskonforme Systemveränderung) allgemein äußerst gering zu veranschlagen ist. Als Grund für diese offenbar geringe Krisenlösungskapazität der gegenwärtig etablierten Staatsklasse in abhängigen Ländern kommen zunächst drei Möglichkeiten in Frage: die Staatsklasse ist aufgrund ihrer historisch bedingten Schwäche, der Verfügungsgewalt lediglich über Verwaltungsmittel (im Gegensatz zu Produktionsmitteln, die unter ausländischer Kontrolle bleiben), objektiv zu einer Systemänderung nicht in der Lage; oder sie ist mangels eigener stabiler Klassenbasis im EL eher an einer für sie lukrativen Interessenkoalition mit der metropolitanen Bourgeoisie interessiert, der sie vor allem eine stetige und hohe Profitrate garantieren muß, was eine grundlegende Anderung des neokolonialen Produktionssystems ausschließt; oder ihre geringe Bereitschaft zur Systemveränderung (bei objektiv vorhandenem Handlungsspielraum) ist Resultat eines herrschaftssoziologischen Kalküls, das besagt, daß eine Veränderung des Produktionssystems einem selbstinitiierten Selbstmord als Teil der herrschenden Klasse gleichkommen würde (und somit illusorisch ist). In allen möglichen Fällen des Verhaltens gemäß kollektiven 
Sicherheitsstrebens würde die Staatstätigkeit ein nach innen schwerlich vermittelbares daher krisenpotenzierendes Legitimationsdefizit mit sich bringen. Auch in diesem Sinne könnte man von einem „Ausnahme- oder Notstandsstaat in Permanenz" reden (Sonntag, 1973, 1974).

In jedem Fall ist es angebracht, sich folgende empirisch unbestreitbare Gesetzmäßigkeit der Staatstätigkeit in den heutigen Metropolen (und nur dort?) zu vergegenwärtigen: die permanente Wirtschaftsprogrammierung, Sozial-Regulierung und Krisenantizipation durch einen personell wie finanziell expandierenden Staat (wachsende Staatsquote) ist eine Bestandsvoraussetzung des industriekapitalistischen Profitsystems geworden. Dabei liegt das scheinbare Paradoxon darin, daß mit zunehmender Intervention und Krisensteuerung durch den Staat dieser selbst neue politische und soziale Krisen verursacht bzw. ihr Entstehen mehr oder weniger unausweichlich begünstigt (siehe z. B. die Bürgerinitiativen gegen die staatliche Energiepolitik oder - in der Schweiz - gegen die Entwicklungspolitik) (vgl. B. Guggenberger 1974). Im folgenden wird versucht, eine historisch-logische Rekonstruktion einer bestimmten Staatsform des abhängigen Kapitalismus vorzunehmen. Dabei gehen wir von der Überzeugung aus, daß eine kritische Auseinandersetzung mit den bisher vorgelegten Versuchen zu „Staatsableitungen“ (Altvater, Hirsch, Braunmühl, Basso, Gerstenberger, Flatow/ Hiusken, Blanke/Jürgens/Kastendiek, Hein/Simonis etc.) nicht nur methodisch und heuristisch von Bedeutung für die Analyse des peripheren Staates sein kann, sondern sogar vom Gegenstand her erforderlich ist ${ }^{1}$. Als weitgehend gesicherte Erkenntnis der modernen Imperialismus- und Dependenzforschung kann heute nämlich konstatiert werden, daß die mit der industriellen Revolution in England begonnene Expansion der kapitalistischen Produktionsweise simultan und in wechselseitiger Bedingung das fatale Komplementärprodukt hervorgebracht hat: die Entwicklung der Industriemetropolen einerseits, die gesamtgesellschaftliche Unterentwicklung und strukturelle Abhängigkeit der Kolonien bzw. Peripherien andererseits.

Auszugehen ist daher von der Hypothese, daß es trotz aller Unterschiede in den Produktionsverhältnissen eine gemeinsame ökonomische Basis für beide Staatstypen gibt, die heute in einem weitgehend integrierten Weltmarkt in Erscheinung tritt. Dabei wird die Marxsche Warnung relevant, „daß dieselbe ökonomische Basis“ dieselbe den Hauptbedingungen nach — „durch zahllos verschiedene empirische Umstände, Naturbedingungen, Rassenverhältnisse, von außen wirkende gesellschaftliche Einflüsse usw. unendliche Variationen und Abstufungen in der Erscheinung zeigen kann, die nur durch Analyse dieser empirisch gegebenen Umstände zu begreifen sind“. Mit dieser Aufforderung zur empirischen Analyse historisch-geographischer „Abstufungen“ und „Variationen“ endet der Abschnitt im dritten Band des „Kapitals“, der die vielzitierte Passage enthält: „Es ist jedesmal das unmittelbare Verhältnis der Eigentümer der Produktionsbedingungen zu den unmittelbaren Produzenten . . ., worin wir das innerste Geheimnis, die verborgene Grundlage der ganzen gesellschaftlichen Konstruktion und daher auch der politischen Form des Souveränitätsund Abhängigkeitsverhältnisses, kurz, der jedesmaligen spezifischen Staatsform finden“. (MEW 25, 1969, 799/800). Für die EL stellt sich demnach die Frage: wer ist der Eigentümer der Produktionsbedingungen (nicht nur der Produktionsmittel, die

\footnotetext{
1 Die Auseinandersetzung des Autors mit dieser Ableitungsdiskussion kann hier explizit nicht erfolgen, sie liegt aber manchen hier vorgetragenen Ưberlegungen zugrunde. In diesem Zusammenhang ist vor allem der Essay von Tilman Evers nützlich für die Schärfung des Problembewußtseins.
} 
weitgehend verstaatlicht worden sind). Dabei wird dies „geheimnisvolle“ Verhältnis an gleicher Stelle historisch-dialektisch definiert als ein Verhältnis, „dessen jedesmalige Form stets naturgemäß einer bestimmten Entwicklungsstufe der Art und Weise der Arbeit und daher ihrer gesellschaftlichen Produktivkraft entspricht" (799). Historischempirische Analysen von Gesellschaften mit struktureller Heterogenität der sozialen und ökonomischen Verhältnisse - einer eminent historischen Kategorie - müssen daher mehr sein als „bloße Illustration der jeweiligen Staatsableitungen“ (H. Gerstenberger 1975, 7).

$\mathrm{Da}$ also jeder Staat „ein geschichtlich gewachsenes Gebilde ist, hilft die Klärung seiner Entstehungsgründe, seine Funktionen zu begreifen" (L. Basso, 1975, 14). Dabei tut sich die Notwendigkeit auf, zwei analytische Ebenen desselben historischen Prozesses auch in der Darstellung logisch zu vermitteln, nämlich die genetisch-funktionale Ebene und die herrschaftssoziologische Ebene. Auf ersterer soll die Genesis des Staatsapparates in Schwarzafrika quasi „im historischen Durchschnitt“ erfaßt werden, und zwar als institutioneller und funktionaler Ausdruck eines sich entwickelnden Ausbeutungsverhältnisses (das hier die „von oben“ induzierte Entwicklung von Produktivkräften voraussetzt und bewirkt). Der internationale historische Rahmen ist dabei die gewaltsame Unterwerfung und Integration der Peripherien (Kolonien) in einen Weltmarkt, der vom Konkurrenzkampf imperialistischer Mächte strukturiert und beherrscht wurde. Die zweite analytische Ebene behandelt Funktionsweise und Bewegungsform des staatlichen Gewaltverhältnisses auf der konkreten Grundlage sich entwickelnder Klassenwidersprüche (als Folge der von außen „aufgeherrschten“ Produktionsverhältnisse), d. h. ein Herrschaftsverhältnis (seine politischen und ideologischen Vermittlungsinstanzen, seine Integrations- und Repressionsmittel und -kosten). Das Zusammen- und Ineinanderwirken beider Dominanzverhältnisse im Verlaufe von 80 Jahren Kolonialherrschaft formte eine Zentrum-Peripherie-Struktur, die auch dem postkolonialen Staat, bzw. der jeweiligen Staatsklasse, wenigstens zunächst Aktionsradius wie Handlungsschranken vorzeichnete: m. a. W. den Handlungsspielraum für (a) Eingriffe in den lokalen Reproduktionsprozeß und (b) für Eingriffe in die sozialen Beziehungen zwischen den einheimischen Produzenten und den „Eigentümern der Produktionsbedingungen“, d. h. hier der Staatsklasse plus metropolitaner Bourgeoisie. Die Geschichte dieser versuchten, versäumten, unmöglichen und tatsächlich erfolgten Eingriffe der jeweiligen „Staatsklasse“ bildet im Kern die Geschichte der neuen Staaten Afrikas.

\section{Die historische Herausbildung von Staatsfunktionen und Klassenformationen in Afrika zwischen 1880 und ca. 1960}

Wenn es richtig ist, daß bei jeder Analyse (des Klassencharakters und der Handlungsspielräume eines kapitalistischen Staates) die historisch geprägte Staatsform vor allem aus den materiellen Lebensverhältnissen der betreffenden Gesellschaft abzuleiten ist, dann legitimiert sich eine Vorgehensweise, die das Werden von Staatsfunktionen im Zentrum und in der Peripherie als einen zusammenhängenden Geschichtsprozeß begreift. Im folgenden wird daher ein methodisches Verfahren angewandt, das aus der historisch-logischen Rekonstruktion der abhängigen Entwicklung einer begrenzten Peripherieregion (Schwarzafrika) zwei Seiten eines kapitalistischen 
Expansionsprozesses (zwischen 1880 und 1975) zu bestimmen versucht ${ }^{2}$ : die simultane und wechselseitig sich bedingende Herausbildung von peripheren Staatsfunktionen und abhängigen „Klassen“-Formationen, wobei die Konkretisierung des Klassenbegriffs besondere Schwierigkeiten (s. u.) mit sich bringt. Die „new states“ Afrikas verdanken, geschichtlich betrachtet, ihre Existenz zunächst einmal den bürgerlichen Nationalstaaten des Europas im 19. Jahrhundert. Buchstäblich in Ausübung der "Weltmarktfunktion" des bürgerlichen Staates (s. u.) im Zeitalter imperialistischer Konkurrenz wird das angeblich "herrenlose“ Land in Afrika, den spezifischen Bedürfnissen der jeweiligen Metropole entsprechend, in Besitz genommen und samt seiner rechtlosen Einwohner, verwertet'. Je nach den diversen Akkumulationsbedingungen, den Wachstumsstörungen und sozialen Spannungen im Inneren einer Metropole kamen die imperialistischen Staatsbürokratien diesem historischen Imperativ nach. Dabei handelte es sich hauptsächlich um die Beschaffung und Sicherung folgender außereuropäischer Produktionsfaktoren und Absatzbedingungen, die für die sich rasch erweiternde Reproduktion der Industriegesellschaften notwendig waren:

- mineralische Rohstoffe (Eisen, Kupfer, Gold, Öl etc.),

- tropische Handelsgewächse als Rohstoffe für neue Industriezweige (Baumwolle, Kautschuk) und neue Konsumbedürfnisse (Kaffee, Tee, Tabak etc.),

- Absatzmärkte für industrielle Konsumgüter,

- Land zum Siedeln und/oder

- billige einheimische Arbeitskräfte.

Je nachdem, welche natürlichen Ressourcen, welches kolonialwirtschaftliche Bedürfnis der Metropole und welche agrarische Produktionsform - europäischer Plantagenbetrieb oder afrikanische Familien- oder Dorfwirtschaft - dominierten, entstanden die vier Haupttypen von Kolonial-Territorien in Afrika:

1. die Bergwerk-Kolonie (Typ: Nordrhodesien/Sambia),

2. die Plantagenkolonie (Westafrika) bzw. die

Handelsgewächs-Kolonie (Uganda, Sudan),

3. die Siedler-Kolonie (Algerien, Kenia, Rhodesien),

4. die Arbeitsreserve-Kolonie oder das Arbeiterreservat

(Njassaland/Malawi, Obervolta, Niger).

Der zentrale Vorgang kolonialstaatlicher "In-Wert-Setzung“ („mise en valeur“) bestand darin, die vormals autozentrierten Reproduktionskreisläufe gewaltsam zu unterbrechen und die Existenzfähigkeit der "unmittelbaren Produzenten “ in der Kolonie zu einer abhängigen Variablen der Marktbedürfnisse des „Mutterlandes“ zu machen. Dazu bedurfte es eines bewaffneten Staatsapparates als außerökonomischer Potenz. Da das sich so herausbildende Schema der kolonialwirtschaftlichen Reproduktion und Arbeitsteilung noch heute weitgehend - wenn auch modifiziert und in Erweiterung begriffen - zutrifft, sind auch die heutigen Klassenformationen primär noch aus der geschichtlichen Durchsetzung dieser Produktionsverhältnisse zu bestimmen und zu verstehen - freilich unter Einbeziehung des jeweils besonderen historisch-geographisch-kulturellen Milieus in jedem Peripherieland.

2 Auf literarische Nachweise der kolonialgeschichtlichen Penetration Afrikas wurde hier im allgemeinen verzichtet, vgl. aber Walter Rodney 1972, ferner Hans-Ulrich Wehler (Hrsg.), Imperialismus, Köln und Berlin 1970; Rudolf von Albertini (Hrsg.), Moderne Kolonialgeschichte, Köln und Berlin 1970; Wolfgang J. Mommsen (Hrsg.), Der moderne Imperialismus, Stuttgart etc. 1971; Jean Suret-Canale, Schwarzafrika. Geschichte West- und Zentralafrikas 1900-1945, Band II, Ostberlin 1969; L. H. Gann and Peter Duignan (Ed.), Colonialism in Africa 1870-1960, Vol. 2, Cambridge 1970. 
Mit der unterschiedlich intensiven Integration der Kolonialterritorien in die Weltarbeitsteilung und das heißt auch mit der unterschiedlichen Ausbreitung des Kapitalverhältnisses - am stärksten in den Siedlungskolonien und in den Bergwerksregionen - entstanden kolonialherrschaftlich bedingte Haupt- und Nebenklassen sowie Sozialformationen in Auflösung und im Übergang - Entwicklungsprozesse, die bis heute andauern (vgl. z. B. Herrmann 1973). Für das Verhältnis zwischen Staatsadministration und Gesellschaftsstruktur in den Kolonien war von zentraler Bedeutung, daß hier im Unterschied zu allen autochthonen vorkapitalistischen Gesellschaften (in denen die Gemeinde im allgemeinen Interesse gesellschaftliche Amtstätigkeiten auf Herrschaftsinstanzen übertrug) der imperialistische Staat in der Peripherie erst "seine" Gesellschaft schaffen muß: Mit der Herausbildung der kolonialherrschaftlichen Staatsfunktionen, die nicht primär Ergebnis eigener gesellschaftlicher Auseinandersetzungne in der Kolonie sind, entsteht in und mit den Trümmern der vorkolonialen Gemeinden die sozial heterogene koloniale Klassengesellschaft. Diese ist nicht nur der Etablierung moderner Staatsherrschaft zeitlich nachgeordnet, sondern auch von Anfang an dieser zu- und untergeordnet. In erzwungener Erfüllung des historischen Auftrags seitens des „Mutterlandes“, nämlich die Beschaffung komplementärer Produktionsfaktoren für die rasch sich erweiternde Reproduktion der europäischen Metropolen (Beginn der Elektroindustrie; imperialistisches Wettrüsten etc.) sicherzustellen, bildeten die kolonialen Außenposten der metropolitanen Staatsapparate (die direkten ,Vorläufer' der heutigen staatlichen Technostruktur in den EL) in der Peripherie folgende Staatsfunktionen und Gesellschaftsformationen heraus $^{3}$ :

1. Die historisch erste Aufgabe war die militärische Eroberung und völkerrechtliche Sicherung des Territoriums gegen die Ansprüche konkurrierender Kolonialmächte. Die "Schutzgebiete“ wurden mit befestigten Verwaltungsstationen, Militär- und Polizeitruppen ausgestattet. Darin manifestierte sich die sog. „Weltmarktfunktion“ des bürgerlich-imperialistischen Staates aufs deutlichste.

2. Nach Etablierung der Grundstruktur für einen staatlichen Repressions- und Ordnungsapparat folgte die Einführung einer europa-zentrischen Rechtssprechung, die die einheimischen Rechtsnormen in unterschiedlichem Maße verdrängte und überlagerte. Ihre Funktion bestand vor allem (a) in der schein-legalen Aneignung von "herrenlosem" Land (Einführung des Rechts auf individuellen Bodenbesitz, das kollektive Wirtschaftsformen auflöste), (b) mittels der Kopfsteuer-Gesetzgebung in der Aneignung von (vorkapitalistisch produziertem) Mehrprodukt und (c) in der administrativen Einrichtung eines Arbeitsmarktes oder einer Arbeitsgesetzgebung, die reinen Zwangscharakter trug (Verpflichtung der "Eingeborenen“ zur Zwangsarbeit auf Plantagen, zur Fronarbeit, zum Wegebau etc.). Auf diese Weise schafft die Kolonialadministration aus einer bäuerlichen Selbstversorgungswirtschaft die erste und wichtigste „Klasse“ (s. u.): den Kontrakt- oder Wanderarbeiter als „unmittelbaren Produzenten" in Bergwerken und Plantagen. Es handelt sich um den zwangsrekrutierten Proletarier auf Zeit. Während in den Siedlerkolonien und oft auch in den Bergwerkskolonien die Proletarisierung der Beschäftigten weitgehend vollständig durchgeführt wird - d. h. die landlos gemachten Arbeiter leben nur noch vom

\footnotetext{
3 Die folgenden Ausführungen gelten nicht für die Siedlerkolonien, die einer eigenen - von den weißen Siedlern dominierten - Entwicklungsdynamik unterworfen waren bzw. noch sind. Siehe dazu Kenneth Good, Settler Colonialism: Economic Development and Class Formation, in: The Journal of Modern African Studies, Vol. 14, Nr. 4 (1976), S. 597-620. Darin untersucht Good die Verhältnisse in Kenia, Algerien, Rhodesien und Südafrika.
} 
Verkauf ihrer Arbeitskraft - , bleibt in den anderen Kolonien der halbproletarisierte Kontrakt- oder Wanderarbeiter als Klassentyp vorherrschend: er besitzt noch bäuerliche Subsistenzmittel (ein Minifundium), aber nicht mehr genug, um so das ganze Jahr über seine Familienexistenz zu reproduzieren. Ihn zwingen Steuer, Gesetze und nackte Not zum zeitweisen Verkauf seiner Arbeitskraft, während eine permanente Nachfrage nach seiner nichtqualifizierten Arbeitskraft (aus ökonomischen und herrschaftssichernden Gründen — Kolonialstädte sollten ,weiße' Städte bleiben -) nicht vorhanden ist. Prozesse der ursprünglichen Akkumulation von Kapital wurden somit eingeleitet - Trennung der Produzenten von ihren Produktionsmitteln - aber auch frühzeitig blockiert.

Hier manifestiert sich jenes peripher-kapitalistische Phänomen der Auflösung/Erhaltung von Produktionsverhältnissen sehr deutlich. Dieser Zustand ist bis heute eine Hauptschranke für die volle Durchsetzung des Kapitalverhältnisses in den Peripherien geblieben. Dies erklärt sich wohl zum Teil daraus, daß er eine Voraussetzung für die ruinöse Niedrighaltung der Löhne (der Reproduktionskosten) und damit für die Produktion von kolonialen Extraprofiten war und (teilweise) noch ist (dazu D. Senghaas 1974).

3. Mit zunehmender Nachfrage nach industriellen Rohstoffen und tropischen Genußmitteln in den Metropolen wurden die Kolonialverwaltungen aus Konkurrenzgründen gezwungen, „allgemeine Produktionsbedingungen" für einen systematischen Anbau bzw. Abbau von Kolonialwaren zu schaffen. Mittels Krediten, „Schutzgebietsanleihen ", Reichsgarantien und der Entsendung von technischem und wissenschaftlichem Personal schuf die Kolonialadministration erstmalig materielle Produktionsvoraussetzungen für eine Marktproduktion. Dabei spielen Investitionen im Infrastrukturbereich, vor allem im Verkehrssektor, eine zentrale Rolle - quasi als Pionierprojekte. So entstehen in den zwei Jahrzehnten vor dem 1. Weltkrieg überall in Afrika Überlandstraßen und Eisenbahnen, und zwar von den neu gebauten oder erweiterten Häfen ins Landesinnere, zu den Bergwerken, Plantagen, den Arbeitsmärkten sowie zu den Sammelstellen von "Cash crops" aus den landwirtschaftlichen Monokulturgebieten.

Das primäre Ziel dieser kolonialherrschaftlichen Maßnahmen war meistens die Aneignung von industriellen Rohstoffen oder aber die reale oder eingebildete Stärkung der Weltmarktposition der jeweiligen Metropole; ihr Ergebnis war jedenfalls die zunehmende Integration der Peripherien in eine ungleiche vertikale Arbeitsteilung zwischen der Kolonialmetropole und ihren Besitzungen in Übersee.

4. Mit dem $Z_{\text {wang }} \mathrm{zu}$ wachsender Arbeitsteilung innerhalb der kolonial beherrschten Peripherie-Gesellschaft, mit dem die Ausweitung des Geld- und Warenverkehrs Hand in Hand geht, erfordert es das „allgemeine Interesse“ (der Siedler, Kaufleute sowie der Exportbourgeoisie der Metropolen), daß auch die soziale Infrastruktur den Verwertungsbedürfnissen des metropolitanen Kapitals angepaßt wurde. So entstand vor allem ein ausbeutungs-funktionales, höchst selektives formales Schul- und Ausbildungssystem, das im Laufe der Zeit folgende Berufsfunktionäre auf unterer Stufe hervorbrachte:
- Soldaten, Polizisten und Zöllner;
- Lehrer, Missionskatecheten und Dolmetscher;
- Schreibkräfte, Buchhalter und administrative Hilfskräfte aller Art;
- schließlich Hauspersonal, Krankenhelfer, „Boys“ aller Art. 
Mit der Ausübung dieser Infrastrukturfunktionen zur Absicherung von kolonialer Ausbeutung und Herrschaft schuf der koloniale Staat eine extrem einseitige Erwerbsbevölkerung, genauer gesagt: die Grundlagen für eine dienstleistende Klassenstruktur. Die Heranbildung von nur administrativen Kadern bei gleichzeitiger Verhinderung der Ausbildung $\mathrm{zu}$ technischen und kommerziellen Berufen (als potentiell günstige Rekrutierungsbasis für eine einheimische Industriebourgeoisie) gibt der kolonialen (und postkolonialen) Klassengesellschaft ihr bürokratisches Gepräge. Die strukturelle Ursache dafür ist freilich darin $\mathrm{zu}$ suchen, daß die asymmetrische Arbeitsteilung zwischen Metropole und Kolonie höchstens die staatlich reglementierte Entwicklung eines kolonialen Agrarkapitalismus (mit zu- und untergeordneter Restsubsistenzwirtschaft) zuließ. Dieser hatte zudem die Besonderheit, daß aller vermarktbare Surplus - der normalerweise als Profit die Existenzgrundlage der Bourgeoisie sichert außer Landes gebracht wurde (und somit nicht reinvestiert werden konnte) (vgl. R. Dumont 1967 und S. Amin 1975).

Diese Politik der rigorosen Abschöpfung wurde erst in dem Augenblick modifiziert (meist nach 1945), als die europäischen Kolonialadministrationen ein Herrschaftsinteresse daran zeigten, „progressive Farmer" unter den Einheimischen heranzuzüchten, um die politische und soziale Ordnung auf dem Lande zu stabilisieren, die von einem landlos gemachten oder überausgebeuteten Bauerntum bedroht wurde (wie z. B. in Kenia). So entstand in den Cash-crop-Gebieten sowie in den Regionen, in denen Nahrungsmittel für einheimische Märkte angebaut werden konnten, allmählich eine Schicht von „Kulaken-Bauern“ (s. u.). Diese hatten bald mehr als ihre Ketten zu verlieren und waren auf Anstellung billiger Lohnarbeiter angewiesen. Damit war bewußt ein sozialer Differenzierungsprozeß auf dem Lande in Gang gebracht worden, der staatlicherseits gefördert wurde (nur progressive Farmer erhielten Kredite, Marktzugang, Beratung etc.). Er ließ die von außen penetrierten afrikanischen Agrarwirtschaften zu einem hierarchischen vielschichtigen Ausbeutungssystem werden, in dem vorkoloniale Dienstleistungsmethoden mit kapitalistischer Marktgesetzlichkeit auf unterschiedlichste Weise verschmolzen (strukturelle Heterogenität der Produktionsverhältnisse).

5. Für die kolonialen Peripherien wurde die Enklavenproduktion die kennzeichnende Form außenabhängiger und exportspezialisierter Kolonialwirtschaft. Sie ließ keinen inneren Markt entstehen, was schwerwiegende desintegrierende Folgen für die Gesellschaft und ihre Subsistenzwirtschaft hatte. Die Enklavenproduktion - die nach reinen Kostenkriterien und ohne soziale Rücksichten nur dort Güter herstellt, wo sie am billigsten zu beschaffen sind und eine besondere Form „ungleicher Entwicklung“ darstellt - fand ihre politische Entsprechung oder Ergänzung im Herrschaftsprinzip des "divide et impera“:

Die Rekrutierung sowie die ausbeutungs-funktionale Ausbildung von administrativen Hilfskadern erfolgte auf der Basis ethnischer und regionaler Unterschiede: je segmentierter ein Volk, desto widerstandsschwächer und somit brauchbarer war es im allgemeinen für den Kolonialherren. Neben den so erzielten Kosteneinsparungen für Herrschaftssicherung sollte erreicht werden, daß sich über die Zuspitzung und Ausdifferenzierung regionaler und kultureller Unterschiede weder ein regionalübergreifendes antikoloniales (nationales) Klassenbewußtsein entwickeln könnte, und dies weder auf der Ebene der Lohnarbeiter, noch auf der der einheimischen Pflanzerbourgeoisie (wo diese überhaupt entstehen konnte). 
Die ökonomische (Enklavenproduktion) und soziale (ethnisch separierte Berufsrekrutierung) Isolierung der Regionen und Wirtschaftssektoren untereinander verstärkte bestehende vertikale Identitätsorientierungen der ethnisch differenzierten Bevölkerung. Auf diese Weise konnte der „Tribalismus“ nun auch als Folge der ungleichen Entwicklung im Kolonialsystem seine große politische Bedeutung erlangen. Mit ihm war nämlich die Grundlage für eine Struktur von Machtkampf gelegt worden, der wohl in einzigartiger Weise die postkolonialen Gesellschaften Afrikas (auch stärker als in Asien) prägte: anstelle antagonistischer Klassen, die um alternative Gesellschaftsordnungen gegeneinander kämpften, rivalisierten hier vor allem regional und meist ethnisch kohärente soziale Gruppen, die unterschiedliche Wirtschaftssektoren repräsentierten, um die politische Hegemonie über die staatliche Ressourcenzuteilung (s. u.).

Zahlreiche jüngere Untersuchungen haben zutage gefördert, daß auch heute noch die Mobilisierung ethnischer Loyalitäts- und Identitätsgefühle für die Konsolidierung oder Erlangung politischer Anteile an der Staatsmacht von einzelnen Machtaspiranten skrupellos mißbraucht wird, mit bemerkenswerten Folgen der Systemimmunisierung. So konstatierte z. B. Claude Ake, daß vor allem „ethnische Identität“ als Instrument im politischen Verteilungskampf „ein wirksames Gegenmittel gegen die Entwicklung von Klassenbewußtsein“ sei. Es schaffe „vertikale Beziehungen über Klassenschichten hinweg und helfe dem politischen System, ein Integrationsniveau aufrechtzuerhalten, das weit über den objektiv vorhandenen Klassendifferenzen liegt" (Ake 1976, 9).

6. Wenn auch die kolonialen Staatsadministrationen die Herausbildung einer Produktionsmittel besitzenden und akkumulationsfähigen einheimischen Kolonialbourgeoisie zu verhindern suchten und im allgemeinen auch Erfolg dabei hatten (regionale Ausnahme: die Pflanzerbourgeoisie in Handelsgewächskolonien, die auf keine europäische Konkurrenz stieß, wie die Kakao-Farmer in Ghana), so gestattete der zunehmende Warenhandel in der Kolonie berufliche und soziale Aufstiegschancen in der Zirkulationssphäre. Überall entstanden die kolonialen „Zwischenschichten“:

- Ernteaufkäufer, rurale Ladenbesitzer und Geldleiher;

- Wanderhändler und städtische Kramladenbesitzer,

— die berühmten „market women“ der westafrikanischen Städte,

- in Kommission für europäische Im- und Exporteure agierende Geschäftsleute im Zwischenhandel - die Nachfolger vorkolonialer "merchant princes“ (vgl. K. Y. Daaku 1970) und

- mit der Entwicklung der staatlichen ,Marketing Boards ${ }^{c}$ - der Vermittlungsinstanz zwischen Bauern und Zentraladministration - Fuhrunternehmer und andere Unternehmer von Dienstleistungsbetrieben.

Mit zunehmendem Warenaustausch zwischen Stadt und Land, Küste und Hinterland, Bauern und Konsumwarenhändlern - m. a. W. mit der Ausbreitung des Kapitalverhältnisses im restriktiven kolonialen Rahmen - entstand die „kommerzielle Bourgeoisie“. Sie war dadurch gekennzeichnet, daß sie sich auch ethnisch-kulturell von den "unmittelbaren Produzenten“ unterschied. Waren es in Ostafrika die ethnischen Minderheiten der Inder, so in Westafrika vornehmlich die der Syrer und Libanesen, die sich im Kielwasser des europäischen Kolonialismus feste ökonomische Positionen sicherten. Nach der Unabhängigkeit richtete sich die erste Runde im afrikanischen Klassenkampf zwangsläufig gegen diese Minderheiten, die wesentlich zum Funktionieren des kolonialwirtschaftlichen Ausbeutungssystems beigetragen hatten (vgl. vor allem I. Shivji 1975). Aber nicht auf dem Wege der Verdrängungskonkur- 
renz, sondern auf dem bürokratisch-administrativen Wege der sozialen Enterbung und Rollenzuweisung wurde hier der "stille“ Klassenkampf ausgetragen. Er nahm meist die Form staatlicher Afrikanisierungspolitik an, mit mehr oder weniger existenzgefährdenden Folgen für die verdrängte kommerzielle Bourgeoisie.

7. Nicht zu übersehen schließlich ist die Tatsache, daß die direkte formelle Kolonialherrschaft der imperialistischen Metropolen einem besonderen Legitimationszwang ausgesetzt war: zum einen als kostenverursachende Staatstätigkeit gegenüber der nationalen Opposition in der Metropole (den europäischen Arbeiterparteien), zum anderen als rassistische Fremdherrschaft gegenüber der allmählich politisch bewußter werdenden akkulturierten einheimischen Bildungseliten (der sog. „Intelligentsia“ oder „Honoratioren-Elite"), die am meisten unter der rassischen Diskriminierung der Europäer zu leiden hatten (vgl. G. Grohs 1967). In diesem politischen Kontext produzieren die an der Aufrechterhaltung des Kolonialismus interessierten großbürgerlichen Kreise (expansionistische Industriegruppen etc.) besondere Rechtfertigungsideologien, die - unbeabsichtigt, aber unvermeidlich - auf Bewußtsein und Verhaltensweise der sozio-kulturell entwurzelten Afrikaner von bestimmendem Einfluß werden sollte (und mindestens die erste Generation nach der Unabhängigkeit noch prägen). Dies ereignete sich im doppelten und widersprüchlichen Sinne: Einmal verstärkten die kolonialen Herr-Knecht-Ideologien („Assimilation“, „Indirect Rule“, wohlwollender Paternalismus, Erziehung zur Arbeit etc.) das Minderwertigkeitsgefühl der nicht-assimilierbaren und diskriminierten Kolonialuntertanen (mit all den von Franz Fanon beschriebenen psycho-kulturellen und politischen Folgen); andererseits stellten die Kolonialherrschaft vermittelnden Zivilisations-Institutionen wie Schule, Kirche, Mission und Bürokratie mit ihren entsprechenden "Werten" (Gleichheit vor dem Gesetz, Freiheit der Wirtschaftssubjekte, christliche Brüderlichkeit, demokratisches Wahlrecht etc.) einer anpassungsfähigen Minderheit auch die Instrumente bereit, mit deren Hilfe sie kulturellen "Anschluß" und politische Partizipation (z. B. im Legislative Council der englischen Kolonien) fordern konnten. Der Preis dafür war eine zwangsläufige Entfremdung dieser Eliten von ihrem einheimischen Milieu, nicht nur in ihrem Bewußtsein, sondern auch in bezug auf die materielle Basis ihrer Existenz. Sie erlangten strategische Bedeutung, weil sie sich als einzige soziale Gruppen im Rahmen der kolonialherrschaftlichen Spielregeln als nationalistische Avantgarde politisch zu artikulieren vermochten (R. v. Albertini 1966, Grohs 1967).

Was sich im Afrika der fünfziger und Anfang der sechziger Jahre abspielte, war bekanntlich nicht eine soziale Revolution, sondern ein ziemlich geordneter "Transfer of power" unter Lizenz der Kolonialmetropolen. Er vollzog sich in wohl kontrollierten Etappen (im Gegensatz zum revolutionären Durchbruch in Algerien oder Mozambique): Machtbeteiligung, interne Selbstverwaltung und schließlich volle staatliche Souveränität auf der Grundlage einer genehmigten Verfassung. Dieser Anpassungsprozeß sollte den Beweis der Regierungsfähigkeit der afrikanischen „Intelligentia" erbringen - und zwar gegenüber den fremden Kolonialherren in der Metropole. Genau darin bestand der Geburtsfehler der ersten afrikanischen "Staatsklasse“. Sie bezogen ihre politische Legimität mehr durch die Kooperation mit der Metropole, wie konfliktreich diese im einzelnen auch sein mochte, als durch Konfrontation an der Spitze einer klassenkämpferischen Bewegung gegen ökonomische Ausbeutung. Dies war eher die Funktion der militanten gewerkschaftlich organisierten städtischen Lohnarbeiter, die gegenüber einer nationalistischen Befreiungsbewegung, die sich im wesentlichen auf den anti-kolonialen Protest der bäuerlichen Massen stützte, meistens 
eine ambivalente Haltung einnahmen: konnte es im langfristigen Interesse der städtischen Arbeiterklasse sein, wenn nun mit Erlangung der politischen Unabhängigkeit afrikanische Bürokratie-Eliten den kolonialen Staatsapparat usurpierten, gegen den die Arbeiter jahrzehntelang angegangen waren?

\section{Die Usurpation des kolonialen Staatsaparates durch die afrikanische „Petty Bourgeoisie“}

Diese (knappe und zweifellos vergröbernde) historische Rekonstruktion von Genesis und Funktion des kolonialen Staates in Afrika hat folgende Zusammenhänge aufgezeigt, die für das Verständnis der nachkolonialen Gesellschaftsstrukturen und Herrschaftsverhältnisse u. E. grundlegend sind:

1. Im Unterschied zum bürgerlichen Staat Europas, der sich beim Übergang vom Feudalismus zum Kapitalismus als "merkantile Außenvertretung" (H. Gerstenberger 1973) im wesentlichen innergesellschaftlich herausbildet, verdankt der peripher-kapitalistische Staat Afrikas seine Entstehung einem exogenen Gewaltakt, der imperialistischen Expansion des europäischen Industriekapitalismus zwischen 1880 und 1960. $\mathrm{Er}$ ist primär ein exterritorialer Ableger der miteinander konkurrierenden metropolitanen Staaten auf der Suche nach Rohstoffen und/oder sicheren Weltmarktund Weltmacht-Positionen.

2. Die historische Mission des kolonialen Staatsapparates in Afrika war die zwangsweise Durchsetzung der kapitalistischen Produktionsweise im besonderen Interesse einer Kolonialmetropole. Er wirkt dabei als Agent der Zersetzung vorkapitalistischer Produktionsverhältnisse. Seine Hauptfunktion besteht darin, die außerökonomische Proletarisierung von Subsistenzbauern einzuleiten und sicherzustellen. Die diesen Vorgang bewirkenden Herrschaftsmittel waren (a) Zwangsarbeit, (b) Steuer und materielle Anreize, (c) Schulausbildung und soziale wie auch Einkommensprivilegien.

3. Der koloniale Ablegerstaat als Garant der imperialistischen Interessen der Metropole wirkte als Hebamme einer qualitativ neuen, wenn auch strukturell heterogenen Gesellschaft. Er erfüllt seine historische Mission, indem er

a) Ruhe und Ordnung zum Zwecke direkter und indirekter Ausbeutung schafft: es entstehen Polizei, Armee, Richter, Steuereinzieher etc.,

b) allgemeine Produktionsvoraussetzungen für enklavenartige Exportproduktion schafft: es entsteht das Proletariat im Dienstleistungssektor, Eisenbahn- und Hafenarbeiter etc.,

c) verwaltet und ausbildet im Interesse kostengünstiger Herrschaftsausübung: es entstehen Bürokraten, Verwaltungsangestellte und Lehrer,

d) vermarktbares Mehrprodukt einsammelt, abschöpft und (in die Metropole) transferiert und gleichzeitig für die Ausbreitung von Geld- und Warenverkehr sorgt: es entstehen Händler, Geldleiher, Handwerker, kurz kommerzielle Zwischenschichten, und indem er

e) im Sinne der ursprünglichen Akkumulation die bäuerlichen Produzenten von ihren Produktionsmitteln trennt (in den Siedler- und Bergwerkskolonien) und diese (Land, Rohstoffe) der externen Bourgeoisie zuschanzt oder aber (im Fall der Handelsgewächskolonien) eine einheimische Klasse von Landbesitzern schafft (P. Hill 1970).

Im Gegensatz zur allmählichen Durchkapitalisierung der Metropolen bringt der extern gesteuerte, abhängige Entwicklungsprozeß in den Peripherien keine vollwertige 
lokale Bourgeoisie hervor, d. h. eine Klasse, die die nationalen Produktionsmittel als Privateigentum besitzt, Kapital akkumuliert und reinvestiert und sich die Staatsmacht dienstbar macht. An dieser Stelle wird es notwendig, den Klas s e n b e g r if f für afrikanische Gesellschaften zu erörtern und zu operationalisieren. Die Tatsache, daß sich heute das wissenschaftliche und politische Hauptinteresse auf jene diffus erscheinende "Klasse" richtet, die in verwirrender Vielfalt als "neue Elite“ (P. Lloyd) als „Strategische Eliten (Bryde), als „bürokratisch-militärische Oligarchie“ (H. Alawi), als „nicht-kapitalistische“ oder kleinbürgerliche oder bürokratische Staats-, Nationaloder "Lumpenbourgeoisie“ (A. G. Frank, G. Arrighi, A. Manghezi) bezeichnet wird oder auch schlicht als "herrschende Klasse“ (G. Kitching), ist kaum verwunderlich; denn sie erscheint als das historische Subjekt, das nach siegreichem politischen Dekolonisationsprozeß berufen erscheint, nun auch die ökonomische und kulturelle Entkolonisierung zu erreichen. Gleichzeitig drückt sich in der terminologischen Unsicherheit die anhaltende sozialwissenschaftliche Kontroverse über die Anwendbarkeit des Klassenbegriffs für afrikanische - d. h. hier nicht voll durchkapitalisierte und vor allem auch vertikal segmentierte - Gesellschaften aus.

Dabei sind in der Hauptsache zwei Ansätze zu unterscheiden: während die eher traditionelle Richtung mit Konzeptionen von sozialer Schichtung und herrschenden Eliten operiert (unter Berufung auf G. Mosca, V. Pareto, R. Michels, K. Mannheim), behauptet eine an Marx orientierte, zahlenmäßig zunehmende Gruppe von Sozialwissenschaftlern, daß „die heutigen afrikanischen Gesellschaften nicht nur durch einen hohen Grad an Klassenformation charakterisiert sind, sondern daß die Klassenanalyse der einzig relevante Ansatz für das Studium afrikanischer Gesellschaften sei“ (A. Manghezi 1976, 69). Die traditionelle Schule mit ihren Hauptvertretern Peter Lloyd, S. F. Nadel, M. Kilson etc. definiert die herrschenden „Eliten“ Afrikas häufig in dichothomischer Gegenüberstellung zu den als amorph angesehenen „Massen“ als kleine, aber einflußreiche, sozial homogene, kohäsive und nachahmungswürdige Gruppen. Ihr wichtigstes gemeinsames Kriterium sei moderne intellektuelle Bildung. Dagegen wird besonders auch von afrikanischen Sozialwissenschaftlern das Klassenkonzept für Gesellschaftsanalysen in Afrika bevorzugt. Dazu gehören I. Shivji, S. Amin, A. Manghezi, K. Nkrumah, A. Cabral, E. O. Akeredolu-Ale, W. Rodney, ferner G. Kitching, P. Hill, R. Stavenhagen, R. Ledda, T. Hodgkin, R. First, R. Murray, C. Ake, R. Sandbrook, R. Cohen, R. L. Harris, R. Feldman und andere. Diese Autoren haben in ihren empirischen Arbeiten ernst zu nehmende Einwände gegen das Klassenkonzept wie den folgenden $\mathrm{zu}$ widerlegen versucht: „Während zwar die Elite die Kohäsion, das Bewußtsein der eigenen Privilegien und besondere Lebensstile haben, um den Klassenbegriff zu rechtfertigen, steht ihr jedoch keine andere erkennbare Klasse gegenüber" (P. Lloyd 1967, 315). Den unteren Schichten würde vor allem ein wirkliches Klassenbewußtsein fehlen, was wiederum Folge a) einer relativ hohen sozialen Mobilität von unten nach oben und b) von ethnischen Identitätsmustern sei.

Besonders die Hinweise auf die zunehmenden gewaltsamen Konflikte zwischen städtischen Arbeitern einerseits und der staatlich-parteilichen Verwaltungsbourgeoisie andererseits haben diesen Einwand - mangelndes Klassenbewußtsein - erheblich relativiert, ohne allerdings das Problem der kategorialen Bestimmung von sozialen Klassen in EL aus der Welt schaffen zu können. Hier scheinen (im lateinamerikanischen Konzept entwickelte) „Sektoren-Klassen-Modelle“ zur Konfliktanalyse in Staat und Gesellschaft methodisch fruchtbar zu sein. Sie stellen den Anspruch, horizontale Stra- 
tifikationsstrukturen (Klassen) mit vertikalen, funktional bestimmten Gesellschaftssegmenten und Wirtschaftssektoren verbinden zu können. So beurteilt z. B. Hartmut Grewe Denkanstöße von Markos Mamalakis u. a. wie folgt: „Das hier angeregte Sektoren-Klassen-Modell versucht die Gesellschaft in eine Reihe von ökonomischen Sektoren zu differenzieren, die jeder für sich verschiedene Einkommensgruppen bzw. Klassen beherbergen. Die dabei entstehende Matrix dient als Taxonomie der wichtigsten Akteure . . . und ist damit bereits ein Fortschritt verglichen mit dem klassischen drei- bis fünfschichtigen Klassenkonfliktmodell ... Die vortheoretische Annahme dieses Ansatzes besagt, daß mit seiner Hilfe das Forschungsinteresse sowohl auf die horizontalen Tauschprozesse und Tauschmuster der Ressourcenkonkurrenz ökonomischer Sektoren als auch auf die vertikalen, verhaltensmäßig bedingten Tauschbeziehungen und Kooperationsvereinbarungen von verschiedenen Status- bzw. Einkommensgruppen ein und desselben Produktionssektors gelenkt wird“ (1974, 29).

Das Problem bei diesem Ansatz besteht nun freilich darin, im Einzelfall herauszufinden, welcher der beiden konfligierenden Tauschprozesse, von denen eine Gruppe einer sektoralen Produkionsgemeinschaft entsprechende Einkommensvorteile erwartet, für diese Priorität hat. Auch Grewe verzeichnet die „Tatsache, daß zwischen sektoralen Mitgliedern, die verschiedenen Einkommens- bzw. Prestigegruppen angehören, enorme Spannungen bestehen können" $(1974,29)$.

Trotz dieser Schwierigkeiten läßt sich u. E. dieses Konzept auch für den staatlichen Sektor operationalisieren; denn in der Praxis der afrikanischen Länder greift die Staatsklasse oft direkt oder indirekt auch in den Konkurrenzkampf zwischen Produktionsgemeinschaften bzw. Wirtschaftssektoren ein (z. B. zwischen Exportlandwirtschaft und Konsumgüterindustrie), u. zw. auf dem Wege der Revenueverteilungspolitik. Darüber hinaus kann die Hypothese aufgestellt werden, daß dort, wo die Regierung selbst zu einem eigenen Wirtschaftssektor wird (mittels „parastatals“, s. u.), mit wachsender Verfügung über Ressourcen sich die Interessengemeinschaft Staatsbürokraten zu einer „bürokratischen Staatsbourgeoisie“ mit eigenem (horizontalen) Klassenbewußtsein mausert ${ }^{4}$.

Für die Konfliktanalyse der afrikanischen Gesellschaften der Gegenwart soll daher jetzt eine Definition von Klasse vorgenommen werden, die den allgemeinen Aspekt der kapitalistischen Gesellschaft (die organisierte private Aneignung von fremder Mehrarbeit als Ware) mit dem historisch besonderen Aspekt einer strukturell abhängigen und vertikal stark segmentierten Entwicklungsgesellschaft verbindet. Die Bezeichnung Klassengesellschaft für afrikanische Verhältnisse läßt sich rechtfertigen aufgrund der Tatsache, daß heute erstens die Mehrheit der Bevölkerung aus voll oder partiell proletarisierten Produzenten für den kapitalistischen Markt besteht und daß zweitens sich die herrschenden und regierenden sozialen Gruppen von wie auch immer angeeignetem Mehrprodukt aus verschiedenen Wirtschaftssektoren reproduzieren. Nicht organisiertes Klassenbewußtsein, sozio-ökonomischer Herkunft oder

\footnotetext{
4 Die Bezeichnung „bürokratische Bourgoisie“ für die regierende Klasse ist vielleicht nicht unproblematisch. So macht z. B. H. Elsenhans einen Unterschied zwischen Staatsklasse und Staatsbourgeoisie: "Staatsklasse ist eine Bürokratie, die privilegiert ist und den Produktionsprozeß leitet (!), ohne wie eine Bourgeoisie die Produktionsmittel als Privateigentum zu besitzen ", während er den Begriff Staatsbourgeoisie als irreführend ablehnt, da ,er die spezifischen Probleme der Reproduktion einer nichtbürgerlichen (nicht am Markt konkurrierenden) privilegierten Klasse verdeckt “ $(1976,256)$. Ich verwende den Begriff hier im folgenden Sinne (bezogen auf die ehemaligen Bergbau- und Handelsgewächskolonien): Staatsbourgeoisie oder „bürokratische Bourgeoisie“ - eine im Englischen übliche Bezeichnung (vgl. M. Diop, R. Miller, I. Shivji) meint die lokal regierende Klasse, die sich durchaus, wenn auch vermittelt, am Markt konkurrierend reproduziert, indem sie nämlich mit der metropolitanen Bourgeoisie existentiell verbunden ist und die reproduziert, indem sie nämlich mit der metropolitanen Bourgeoisie existentiell verbunden ist und die (s. u.) hat sie ihre wichtigste materielle Basis.
} 
juristischer Besitz an Produktionsmitteln sind hier die entscheidenden Kriterien für den Klassenbegriff, sondern - soweit es die regierende Klasse angeht - die staatliche Verfügungsgewalt über Produktionsbedingungen und Einkommensverteilung als materielle Basis einer Klassenherrschaft. Demnach kann man die Hauptklassen in abhängig-kapitalistischen Gesellschaftsformationen nach ihrer Stellung und Funktion im Produktions- und Distributionsprozeß als entweder Revenue erzeugende, Revenue verzehrende oder Revenue verteilende Klassen bestimmen. Dabei kann es aufgrund der ungleich entwickelten Wirtschaftssektoren zu diagonalen Interessenbündnissen kommen (z. B. Staatsbürokratie, lokales Unternehmertum und „Arbeiteraristokratie“ versus Landbesitzer und Bauern). Je mehr sich dabei das Bewußtsein von der Ungleichheit der Lebens- und Einkommensverhältnisse zwischen den proletarisierten Klassen und/oder relativ deprivierten Produktionsgemeinschaften einerseits und den herrschenden Klassen andererseits entwickelt - als Begleiterscheinung abhängiger Industrialisierung z. B. - - desto eher ist zu erwarten, daß die Klassenauseinandersetzung von heute noch überwiegend stillen Formen $-d$. h. einerseits staatsbürokratisch repressiven, andererseits sporadisch spontanistischen Formen -, zu offen gewaltsameren und institutionalisierten Formen übergeht (vgl. R. Harris 1975, R. Sandbrook/R. Cohen 1975).

In Anknüpfung an das oben entwickelte Theorem, daß der metropolitane Staat als Hebamme einer strukturellen heterogenen Klassengesellschaft in der afrikanischen Peripherie historisch wirksam war, kann jetzt zwecks genauerer Bestimmung der kolonialen und postkolonialen Klassenformationen in Afrika festgehalten werden: bei aller Würdigung kultureller und regionaler Besonderheiten verdankt jede Klassenformation ihre politische Macht sowie ökonomische Stärke ihrer Zugehörigkeit $\mathrm{zu}$ einem mehr oder weniger produktiven Wirtschaftssektor in Kombination mit ihrer jeweiligen Funktion in einem solchen für die Reproduktion des Staates.

Dabei handelt es sich um die folgenden vier Hauptklassen, die heute je nach Kolonietyp (s. o.) mehr oder weniger deutlich in Erscheinung treten:

1. Die Klasse der ländlichen Lohn-, Wander- und Saisonarbeiter, die - trotz ihrer vielen regionalen Spezifika - ein konstitutives gemeinsames Merkmal aufweisen: zur vollen Reproduktion ihrer Arbeitskraft sind sie partiell auf die ländliche Subsistenzwirtschaft angewiesen. Man könnte diese Klasse als ländliches Halbproletariat bezeichnen.

2. Als entwickeltste Form der proletarisierten Bauern ist ein zahlenmäßig relativ kleines Bergbau- und Industrieproletariat entstanden, das mit zunehmender Industrialisierung (in den Konsumgüter- und Verarbeitungssektoren) dazu tendiert, zu einer urbanen Klasse permanent beschäftigter und klassenbewußter Proletarier europäischen Typs zu werden (s. u.).

3. Die Klasse der ganz oder teilweise für den Markt regelmäßig produzierenden Cash-crop-Bauern. Sie beschäftigen Familienarbeiter und gehen mit der Ausbreitung des Kapitalverhältnisses zur permanenten Anstellung von Lohnarbeitern über. Entwickelte sich in den Handelsgewächs-Kolonien schon frühzeitig eine weltmarktabhängige einheimische Pflanzeroligarchie (z. B. in der Elfenbeinküste und in Ghana), so ist heute die Ausdehnung des Kulaken-Bauerntums (mit zunehmender Landbesitzkonzentration und Lohnarbeiterbeschäftigung) in Schwarzafrika ein weit verbreiteter Vorgang .

5 Siehe die zahlreichen empirischen Belege bei Uma Lele, 1975, und Lionel Cliffe and John S. Saul (Ed.), 
4. Die Klasse der städtischen bürokratischen Kleinbourgeoisie, der „Petty Bourgeoisie". Shivji unterteilt diese am wenigstens klar definierbare Klasse nach Bildung und Beruf in drei Schichten:

- „upper level (Intelligentsia): intellectuals, teachers, higher civil servants etc.,

- middle level: middle government salariat, junior clerks, soldiers etc.,

- lower level: shopkeepers, lower salariat in the services sector etc." $(1975,42)$.

Allerdings ist mit einer solchen formalen Kategorisierung der abhängigen Klassengesellschaft (in Schwarzafrika) noch nicht die politisch zentrale und analytisch kompliziertere Frage nach der herrschenden Klasse - in Abgrenzung zur lokal regierenden Sektor-Klassen-Koalition - beantwortet.

Der Angelpunkt für die Analyse der heutigen Klassenstrukturen in den Staaten Afrikas ist die soziale Zusammensetzung und die politische Orientierung der „Petty Bourgeoisie“, und zwar aus folgenden Gründen: Erstens war sie die einzige lokale embryonale Klasse (bei der Unabhängigkeit), die sich der staatlichen Herrschaftsinstrumente (einschließlich der weißen „expatriates") bedienen konnte und durfte, und zweitens umfaßte sie die Fraktion, die als politisch regierender, aber mittelloser Ausschuß der nationalen Unabhängigkeitsbewegung - als „Pseudo-Bourgeoisie“ (Fanon) - ein objektives Interesse an der Beschaffung einer eigenen materiellen Klassenbasis haben mußte. Dies trieb sie zwangsläufig in Konflikte mit den eigenen Verbündeten aus dem nationalen Unabhängigkeitskampf hinein.

Im Augenblick der Erlangung der formalen Unabhängigkeit - des so heiß ersehnten „political kingdom“ (K. Nkrumah) - sind für diese nationalistische, den kolonialen Staatsapparat rasch usurpierende, Kleinbourgeoisie folgende sozio-kulturellen Merkmale konstitutiv:

a) ein Mindestmaß an westlicher Bildung — was die Degradierung oder Verdrängung vorkolonialer autochthoner Werte beinhaltete,

b) die berufliche funktionale Qualifikation für eine bürokratische dienstleistende Tätigkeit - was ihr den Zugang zu neuen Einkommensquellen ermöglichte,

c) die weitgehende Übernahme urbaner Verhaltensweisen — was die allmähliche Dequalifizierung ländlicher Einstellungen und Lebensweisen bewirkte.

Diese kleinbürgerlich-bürokratischen Gruppen bemächtigten sich eines Staatsapparates, der - wie gezeigt wurde - als das Erbe fremdbestimmter Zweckrationalität zu definieren ist. In der Verfügung der afrikanischen Kleinbourgeoisie hatte der periphere Staat nun aber eine konfliktträchtige Doppelfunktion zu erfüllen: einerseits garantiert er von seiner funktionalen Bestimmung her - als Garant der Exportproduktion - die spezifischen Interessen der metropolitanen Bourgeoisie an sicheren und hohen Profitraten, andererseits erhält er als dankbarer Empfänger von Produktrenten, Royalties und Exportsteuern die Möglichkeit, als monopolartiger Nutznießer und Verteiler von Staatsrevenuen und sozialen Privilegien sich selbst, d. h. der eigentlichen Staatsklasse sowie ihren „supportive classes“ (M. von Freyhold), eine dauerhafte materielle Existenzgrundlage zu schaffen. Eine nationale Bourgeoisie war aus historischen Gründen nicht oder kaum vorhanden. In seinem Buch „Class Struggle

Socialism in Tanzania, Vol. 1 Politics, Vol. 2 Policies, Nairobi 1972 und 1973. Ein „Kulak" ist ein landbesitzender Farmer, der mit Hilfe von Lohnarbeitern Marktprodukte erzeugt ("cash crops“). Er ist zu unterscheiden von reinen Subsistenzbauern (mit Kommunalland), von Genossenschaftsbauern (vom Typ der Ujamaa-Dörfer in Tansania), die eine Beteiligung aller Bauern an den Produktionsmitteln anstreben, aber auch von der traditionellen Agraroligarchie und den "absentee landlords“, die mittels Crop-sharing-Methoauch von der traditionellen Agraroligarchie und den "absentee landlords “, die mittels Crop-sharing-Metho-
den produzieren lassen. Uber die politischen Koalitionen zwischen Kulaken und den lokalen Funktionären siehe z. B. H. U. E. Thoden van Velzen, 1973. 
in Afrika" hat der ghanaische Präsident Kwame Nkrumah die "bourgeoise Situation" der 60er Jahre wie folgt charakterisiert: „Aber obwohl feudale Relikte bleiben, die Kolonialzeit bereitete den kapitalistischen Sozialstrukturen den Weg. Diese Zeit war durch das Entstehen der ,petty bourgeoisie' und einer kleinen aber einflußreichen nationalen Bourgeoisie charakterisiert, die hauptsächlich aus Intellektuellen, ,civil servants', Vertretern akademischer Berufe und aus Offizieren der Streitkräfte und der Polizei bestand. Die Abwesenheit von Kapitalisten unter der Bourgeoisie war auffallend, da lokale Geschäftsunternehmen von der Kolonialmacht im allgemeinen entmutigt wurden . . . Ausländer kontrollierten den Bergbau, die Industriebetriebe, Banken, Großhandel und die großen Farmbetriebe. Tatsächlich war die Bourgeoisie beinahe in ganz Afrika zum großen Teil ,petty bourgeoisie“" $(1970,55 / 56)$.

Die besondere Problemkonstellation beim Übergang zur politischen Unabhängigkeit bestand demnach darin, daß eine (im Werden begriffene und in sich heterogene) „Klasse“ die Staatsmacht usurpierte, die zwar selbst keine oder eine nur sehr schwache, eigenständige ökonomische Basis in der Produktion (als Produktionsmittelbesitzer) hatte, die aber quasi als Substitution für die Möglichkeiten einer Unternehmerklasse über einen Zwangsapparat verfügen lernte, der sich zur mannigfaltigen Aneignung von Produktrenten und Mehrprodukt instrumentalisieren ließ.

Die politische Situation wurde ferner dadurch verkompliziert, daß „power and property “, Staatsmacht und lokales Besitzbürgertum, bürokratische Kleinbourgeoisie und kommerzielle Bourgeoisie fast überall miteinander um Einfluß, Privilegien und Einkommensverteilung rivalisierten. Dies war ein (ererbter) Interessenwiderspruch, der entweder über die Vertreibung (so in Kenia, Uganda und Ghana) oder durch die selektive Kooptation (in Tansania und in Sambia) der kommerziellen Minoritäten mehr schlecht als recht gelöst wurde. Dieser „stille Klassenkampf“ (Shivji) war Teil eines umfassenden Klassenkonsolidierungsprozesses: der Metamorphose der mittellosen aber westlich gebildeten Sektionen der Petty Bourgeoisie im Besitz der Kontrolle über den lokalen Staatsapparat zur entweder nur parasitären Staatsklasse oder zur „Bureaucratic Bourgeoisie“ (M. Diop, R. Dumont, I. Shivji, R. Miller). Ihr Klasseneinkommen in Form von Handelsbeteiligungen, Spekulationsgewinnen, Staatspfründen etc. sind eine direkte Konsequenz ihrer funktionalen Nähe zum staatlichen Entscheidungs- und Verteilungszentrum und nicht umgekehrt. Mit der Konsolidierung dieser Gruppen zur bürokratischen Bourgeoisie oder auch Staatsbourgeoisie - bei gleichzeitiger relativer Deprivation der anderen Fraktionen der Petty Bourgeoisie - ging die Entpolitisierung der Bevölkerung Hand in Hand. Aus der antikolonialen, von den ländlichen Volksmassen unterstützten politischen Bewegung wurde die verfaßte Partei unter Führung der städtischen Intelligenz; aus der Partei, deren Köpfe in die karriere-garantierende Staatsadministration überwechselten, wurde bald ein Hauptstadtclub von politischen Funktionären, der als Rekrutierungsbasis für weitere Regierungsjobs diente. Schließlich hatte die Partei - bzw. das, was von ihr nach dieser personellen Ausblutung noch übriggeblieben war - bis zu ihrer Auflösung oder völligen Entmachtung durch die politischen Bürokraten nur noch folgende Aufgaben wahrzunehmen: (a) rivalisierende Eliten auszuschalten, (b) Akklamationswahlen zu organisieren, (c) eine Abwehrideologie der nationalen und staatlichen Einheit auszuarbeiten, die im schillernden Gewand eines „afrikanen Sozialismus“ die Nichtexistenz von Klassen und Klassenwidersprüchen zu propagieren hat und (d) die Depolitisierung und Zähmung der bäuerlichen Massen und proletarischen Gruppen sicherzustellen. 
Dieser Prozeß verlief je nach Land unterschiedlich und mehr oder weniger turbulent (Staatsstreiche, Militärcoups, Inhaftierung, Exilierung oder Kooptation von Oppositionellen etc.). Dabei war für den unterschiedlichen Grad der politischen Turbulenzen maßgeblich, in welcher Weise sich die bürokratische Bourgeoisie, bestehend aus den höheren militärischen, zivilen und politischen Kadern, mit den drei übrigen Fraktionen der lokalen Bourgeoisie arrangierte (nach R. Ledda), d. h. mit

1. der Kompradoren-Fraktion, „die als Vermittler für die ausländischen ImportExport-Firmen dienen";

2. der lokalen Unternehmer-Fraktion, „die entweder mit dem Auslandskapital assoziiert ist oder aber mit Wohnungsbau, Nahtransport, Kontraktarbeiten etc. beschäftigt ist";

3. der ländlichen Bourgeoisie-Fraktion, die aus „örtlichen Pflanzern, feudalen Landherren und Großfarmern" besteht, „die in der Produktion von Cash Crops für den Export beschäftigt sind“ (R. Ledda, 1967, 565).

Üblicherweise versteht es die Staatsbourgeoisie, enge lukrative Verbindungen mit einer (oder mehreren) Fraktion(en) der lokalen Bourgeoisie zu knüpfen, die wiederum oftmals nur mittels staatlicher Protektion ihre ökonomische Existenz sicherstellen können. Ein Paradebeispiel für solche Trends zur Privatisierung von staatlicher Gewalt bietet Kenia, ein Land, in dem z. B. bis 1971 über 1000 ehemals ausländische Firmen an einheimische Geschäftsleute übereignet werden mußten (Leys 1972 und ILO 1974).

Solche Praktiken schließen keineswegs aus, daß sich oftmals zwischen den verschiedenen (regionalen oder sektoralen) Interessenfraktionen innerhalb des breiten Spektrums der lokalen Bourgeoisie ein erbitterter — wie Ake schreibt „hobbesianischer“ — Positionskampf ereignet. Dabei geht es vordergründig um die Gewinnung der politischen Hegemonie im Staats- und Parteiapparat, der ja ein weitgehendes Distributionsmonopol für Geschäftslizenzen, Kredite und Auslandsverbindungen hat. Langfristig aber muß es vor allem der bürokratischen Bourgeoisie als "Staatsklasse" darum zu tun sein, sich dauerhaft als ökonomisch lebensfähige Klasse zu etablieren und zu konsolidieren, und sei es für den Preis zunehmender externer Abhängigkeit. Dabei ist folgende These von Michaela von Freyhold zu überprüfen: „Der postkoloniale Staat mag seine nationale Bourgeoisie (besser wohl „Petty Bourgeoisie“) unterstützen oder frustrieren oder seine landbesitzenden Klassen oder beide, aber mangels einer Revolution, die die unmittelbaren Produzenten an die Macht brächte, kann er seiner Unterwerfung („servitude“) unter die metropolitane Bourgeoisie nicht entrinnen“ $(1976,7)$.

\section{Die Privatisierung des postkolonialen Staates durch die bürokratische Bourgeoisie}

Für die bürokratische Bourgeoisie gab und gibt es hauptsächlich fünf Möglichkeiten, über die Steigerung der Staatsrevenuen die eigene Klassenbasis materiell zu konsolidieren:

a) Sie weitet quantitativ (flächenmäßig) die Marktproduktion aus und diversifiziert den Export - meist mit Hilfe ausländischer Infrastrukturhilfe;

b) sie erhöht auf stillem Wege die Abschöpfung von Mehrprodukt bei den Marktbauern mittels administrativer Preisregelung (steigende „internal terms of trade“);

c) sie drückt gewaltsam die Mehrwertrate beim Industrieproletariat herauf, dessen Hauptarbeitgeber mit fortschreitender Nationalisierung der Produktion der lokale Staat wird; 
d) sie partizipiert an den Gewinnspannen im Zwischenhandel, erhöht die Steuerund Zollgebühren im Außenhandel oder enterbt auf administrativem Wege die kommerzielle Bourgeoisie (im Zuge der Afrikanisierungspolitik);

e) sie erstreitet auf diplomatischem Wege höhere Zuwendungen aus dem Ausland (Kredite und „Entwicklungshilfe") und bemüht sich (auch kollektiv mit anderen Staatsregierungen) um eine internationale Höherbewertung der jeweiligen nationalen Arbeitsprodukte, d. h. um höhere Rohstoffpreise und günstigere „Terms of Trade" auf dem Weltmarkt.

Zahlreiche Einzelbeobachtungen lassen den Schluß zu, daß die postkolonialen Regierungen Afrikas oft alle fünf Wege der Revenue-Erhöhung und -Aneignung angestrebt haben, je nach Ressourcenausstattung, Weltmarktbedeutung und Prodiktionsverhältnissen des EL. Da sie dabei ganz unterschiedliche Erfolge verbuchen konnten, ergäbe - so ist zu folgern - das je spezifische Mischungsverhältnis der staatlichen Einkommensquellen sowie der Ausgabenbereiche einen vielversprechenden analytischen Ansatzpunkt (Steuer- und Einkommensstatistiken, Preisverhältnisse, Auslandskredite etc.), um so den Klassencharakter der Staatsbürokratie im jeweiligen Land näher bestimmen zu können. Auf der permanenten Suche nach Erhöhung der staatlichen Einnahmen zur Finanzierung a) ihrer eigenen Konsumbedürfnisse und b) der dringend notwendigen Investitionen zur Erweiterung der Produktion entdeckt die Staatsbürokratie schnell die Klasse, die politisch am wenigsten Widerstand leisten kann und sich für administrative Ausbeutung am besten eignet: entweder die bäuerlichen Produzenten und/oder das Bergbau- und Industrieproletariat. Um die Lebenshaltungs- und Reproduktionskosten der städtischen Klassen nach Kräften herabdrücken zu können - bei ohnehin steigender Inflation und Verteuerung aller Importgüter -, wurden fast überall die staatlich administrierten Produzentenpreise für Nahrungsmittel sträflich gesenkt. Diese kurzsichtige Politik resultierte in vielen Fällen in einer bewußten Verweigerung seitens der Bauern, Nahrungsmittel über den eigenen Bedarf hinaus anzubauen. Statt die unterproduktive Landwirtschaft durch staatliche Anreize zu fördern, wurden so Einkommensgefälle zwischen Stadt und Land, aber auch zwischen Markt-Farmern und Subsistenzbauern noch vergrößert, mit all den bekannten Folgen der Landflucht, der Kulakisierung, der steigenden Nahrungsmittelimporte etc.

Allerdings sind die Möglichkeiten für eine bürokratische Staatsklasse, aus Marktbauern und Industriearbeitern ein Mehrprodukt abzupressen, aufgrund der absolut geringen Produktivitätszuwächse schnell erschöpft. Da ferner die Exporteinnahmen fast immer geringer steigen als Bedarf und Kosten von konsumptiven und produktiven Importgütern - wobei letztere erst die Voraussetzungen für die Steigerung von Wachstum und Produktivität schaffen sollen -, ist die zunehmende Staatsverschuldung eine automatische Folge. Der bedrängten Staatsklasse stehen nun zwei Möglichkeiten des Krisenmanagements offen: selektiver Ressourceneinsatz im Innern und Ressourcenbeschaffung von außen. Im ersten Falle wird die sektorale und klassenspezifische Aneignung und Verteilung von Ressourcen zum wichtigsten Thema und gleichzeitig zur bedeutendsten Konfliktquelle staatlichen Handelns. Politik wird primär Allokationspolitik knapper werdender Sektor-Ressourcen; der Staat wird zum Machtzentrum, in dem über Bevorzugung und Benachteiligung von Wirtschaftssektoren und ihren jeweiligen Produzentengemeinschaften entschieden wird. Z. B. entschied sich die ghanaische Staatsklasse unter Nkrumah für die Förderung des städtischen Unternehmertums und gegen die Kakaoproduzenten aller Einkommens- 
schichten (freilich mit unterschiedlichen Graden von Betroffenheit), während die Staatsbürokratie in Tansania im Interesse der genossenschaftlichen Produzenten auf dem Lande bei weitgehender Vernachlässigung industrieller Sektoren agierte, ganz im Unterschied zum Nachbarland Kenia (J. Rweyemamu 1973).

Meistens gleichzeitig wird der zweite Weg des Krisenmanagements beschritten. Nachdem die finanziellen Polster aus der Kolonialzeit mehr oder weniger produktiv aufgezehrt worden waren, wandte sich die Staatsbürokratie in vielen Fällen direkt oder in versteckterer Weise an ausländische Staaten (beider Lager). Die Maximierung der Staatseinnahmen durch Verhandeln mit dem Ausland um mehr Entwicklungskredite, um private Direktinvestitionen, um höhere Exportpreise oder um Schuldennachlaß wurde zur wichtigsten Außenfunktion der regierenden Klasse. Man könnte sie die Revenue-Beschaffungsfunktion nennen, eine Funktion, die proportional zur wachsenden Außenverschuldung an politischer Bedeutung zunimmt. Vor allem hat sie eine wichtige Konsequenz: sie veranlaßt die Staatsbürokratien zu einer permanen ten, wenn auch keineswegs konfliktfreien Entwicklungskooperation mit auswärtigen Staaten. Hier liegen auch wesentliche Gründe dafür, daß die neuen Staaten der Dritten Welt eine internationale diplomatische Aktivität ohnegleichen entfaltet haben: auf bilateral staatlicher Ebene, auf regionaler Ebene (OAU, OCAM, ECOWAS etc.), auf Weltebene (Generalversammlung, UNCTAD, Gruppe der 77 etc.) und nun auch auf der Ebene von Interessenallianzen (Rohstoffkartelle). Der Tatsache Rechnung tragend, daß der Löwenanteil der Staatseinnahmen durch Verwertung der nationalen Produktion auf dem Weltmarkt zustande kommt, streben die Staatsbürokratien danach, ihre abhängigen OOkonomien zu besseren Konditionen in das hierarchische System der internationalen Arbeitsteilung zu integrieren. Dieser im Gang befindliche Integrationsprozeß hat auch auf die lokalen Herrschaftsverhältnisse problematische Konsequenzen: Je mehr die afrikanischen Staatsbürokratien einzeln oder kollektiv ihre politische "bargaining power" gegen die Metropolen einsetzen, um eine günstigere Verteilung der im internationalen Produktionssystem anfallenden Gesamteinnahmen durchzusetzen, desto größer wird die Gefahr, als subordinierte systemintegrierte Klasse von den metropolitanen Zentren existentiell noch abhängiger zu werden und sich somit als „Lumpenbourgeoisie“ eines potentiellen Handlungsspielraums für strukturelle Anderung der Produktionsverhältnisse (in Richtung auf mehr nationale Steuerung der Binnenmarktentwicklung) zu begeben.

Diese Beziehungsstruktur zwischen internationaler (externer) Bourgeoisie in Liaison mit der einheimischen bürokratischen Bourgeoisie einerseits und den ausgebeuteten afrikanischen „Massen“ andererseits hat Issa Shivji aus Tansania in seiner provokanten Analyse der afrikanischen „Neo-Kolonien“ schon 1970 als den Hauptklassenwiderspruch im heutigen Afrika bezeichnet (in „The Silent lass Struggle“) ${ }^{6}$. Da aufgrund der historischen Schwäche der afrikanischen Bourgeoisien eine Austragung von Interessenkonflikten über den ökonomischen Konkurrenzkampf kaum möglich ist, verfestigt sich (trotz aller Höherentwicklung der "gaps“) ein supranationales hierarchisches System sich ergänzender Herrschaftsinteressen. Dabei ist die internationale Bourgeoisie primär an einem günstigen politischen Investitionsklima in den Peripherien interessiert.

\footnotetext{
6 Dieser These ist jedoch mit gewichtigen Einwänden widersprochen worden; sie übersieht vor allem wichtige interne soziale und politische Differenzierungsprozesse, die zu einer Status-quo-Änderung führen können, siehe The Silent Class Struggle, Tanzanian Studies, No. 2, Dar es Salaam 1974.
} 
Aber im Unterschied zum kolonialen Staatsapparat - und dies übersieht Shivji ist heute die lokale Staatsklasse nicht nur von der externen Bourgeoisie der Metropole abhängig, sondern auch - und zwar mit steigenden Erwartungen an den Staat in zunehmendem Maße - von den einheimischen Bourgeoisiefraktionen, die sich im Sog der abhängigen Entwicklung heranbilden bzw. kräftigen: die Dorfbourgeoisie (Kulaken-Bauern), die binnenorientierte Handelsbourgeoisie und die Juniorpartner der großen Auslandsfirmen. Auch sie verlangen von der lokalen bürokratischen Bourgeoisie die Schaffung besonderer Produktions- und Verwertungsbedingungen (Versorgung mit billigen Krediten, Bau von Eisenbahnen, Straßen, Häfen oder von technischen Universitäten etc.). Die Staatsklasse ist somit keineswegs nur „Kompradorenbourgeoisie“.

$\mathrm{Da}$ die bürokratische Bourgeoisie andererseits diese notwendigen Infrastrukturen aus eigener Kraft und mit eigenen Mitteln in der Regel nicht zu erstellen vermag, springen mit eiserner Systemlogik jene internationalen Hilfsagenturen mit staatlichen Ersatz- und Ergänzungsfunktionen in die Bresche, von denen oben die Rede war. Diese historisch erklärliche Unfähigkeit der afrikanischen Staatsbürokratien, sich die allgemeinen Staatsfunktionen einer sich industrialisierenden Gesellschaft selbst voll anzueignen - Konsequenz der Nichtexistenz nationaler Industrie-Bourgeoisien verleitet diese dazu, den von ihren kolonialen Vorgängerinnen beschrittenen Weg der abhängigen außenorientierten Entwicklung forciert fortzusetzen. Hier wird ein Kontinuitätsphänomen erkennbar: die „allgemeinen“ Staatsfunktionen werden (vorübergehend?) von den besonderen Interessen einer externen Bourgeoisie mitbestimmt, die nicht selten als ökonomische „Metamacht" (so D. Senghaas in bezug auf multinationale Konzerne) wirksam wird. Nicht die (angeblich) „klassenlose“ vorkoloniale Gesellschaft wird in den neuen Staaten Afrikas fortentwickelt — wie afrikanische Staats- und Parteiführer der Welt glauben machen wollen -, sondern mit nur wenigen Ausnahmen wird die koloniale (agrarwirtschaftliche) Klassengesellschaft in Richtung auf abhängige Durchkapitalisierung weiterentwickelt: „Tatsächlich ist die Integration der ganzen Ókonomie in das Weltsystem eine der Aufgaben, die der Kolonialismus in verschiedenen Regionen in geringerem oder größerem Maße bewirkt hat. Wo dies in geringerem Maße geschehen ist, da setzten die Regierungen nach der Unabhängigkeit die Aufgaben fort, die der Kolonialismus übriggelassen hat. Sogar die Weltbank würde solche Programme unterstützen, und tut dies auch." (Shivji 1975, 99).

Doch im Unterschied zur Zeit der Kolonialherrschaft ist für den heutigen Zustand hervorzuheben, daß die afrikanische Staatsbourgeoisie mit zunehmender gesellschaftlicher Differenzierung (als Folge neuer Penetrationswellen) in einen explosiven $\mathrm{Zu}$ stand zweiseitiger Belagerung geraten ist: Sie kann meist immer weniger die sozialen Bedürfnisse im nationalen Bereich befriedigen (z. B. im Bildungswesen oder bei der Schaffung von Arbeitsplätzen), noch kann sie den ausländischen Unternehmen dauerhaft freie Hand lassen, will sie sich nicht selbst um jede lokale Legitimation ihrer politischen Herrschaft bringen. In diesem Zustand der Belagerung - schreibt Claude Ake - seien „alle ihre Energien, Ressourcen und Fähigkeiten auf das Überleben gerichtet und auf die Aufrechterhaltung der bestehenden Produktionsverhältnisse“ (1976, 21). Dort, wo die Staatsbourgeoisie den Konflikt zwischen dem (fiktiven) nationalen Allgemeininteresse und ihrem Sonderinteresse als Staatsklasse nicht mehr zu vertuschen vermag, bietet sich letztlich als Ausweg nur noch die Bewaffnung der Staatsklasse an. So wird die atemberaubende Militarisierung der afrikanischen Staa- 
ten zugleich zu einem Maßstab der wachsenden innergesellschaftlichen Widersprüche (vgl. Lloyd 1971, First 1972).

Zusammenfassend läßt sich sagen: Heute befindet sich die bürokratische Bourgeoisie afrikanischer Staaten in einem vielmaschigen Netz neokolonialen Abhängigkeiten, das noch das Produkt historischer Unterentwicklung ist. Je stärker sich dabei die bürokratische Bourgeoisie den übernommenen Staatsapparat als Herrschafts-, aber auch Interventionsinstrument zunutze macht und je unzureichender die verfügbaren Staatseinnahmen, relativ zu den immer steigenden Konsum- und Investitionsanforderungen, desto intensiver wird sie um bilateral nützliche Formen zwischenstaatlicher Entwicklungskooperation im (limitierenden) Rahmen der internationalen Arbeitsteilung zu kämpfen veranlaßt. Die schnelle Ausdehnung des „halbstaatlichen“ Wirtschaftssektors in Schwarzafrika seit den 60er Jahren ist wohl der sichtbarste Beweis für ein abhängiges Entwicklungsmuster, das auf einer transnationalen Interessenallianz zwischen afrikanischer Staatsbürokratie, lokalen abhängigen Bourgeoisiefraktionen und den wirtschaftlich dominanten Auslandsunternehmen beruht.

\section{Staatskapitalismus, „Parastatals“ und wachsende Klassenwidersprüche}

Im Unterschied zum "Staatskapitalismus“ in Industriestaaten - der im Engelschen Sinne nichts anderes ist als die Begrenzung und Regulierung der ökonomischen Spontaneität des Privatkapitals durch den Staat (vgl. Szentes 1974, 92) - verdankt der Staatskapitalismus in Schwarzafrika seine Existenz zunächst der Tatsache, daß eine konkurrenzfähige nationale Bourgeoisie mit der Fähigkeit zur Produktivkraftentwicklung nicht vorhanden ist. Die typische Form, in der dieser historische Mangel kompensiert wird, ist das „gemischte“ Staatsunternehmen oder das „parastatal enterprise“. Der Ausdruck "parastatal“ - so heißt es in einem Regierungsdokument aus Sambia - wird heute weithin akzeptiert „als Bezeichnung des Gebietes nationaler Aktivität, das zwischen dem Civil Service einerseits und dem völlig privaten Unternehmen andererseits liegt . . . Wenn (ein Unternehmen) ein ,parastatal' ist, existiert es dank einer spiziellen Gesetzgebung, die zur Förderung besonderer gouvernementaler Interessen und Ziele geschaffen wurde oder ist, was die Besitzrechte angeht, entweder ganz oder teilweise unter Kontrolle der Regierung" (Report 1975, 130).

Die Besonderheit der Parastatals besteht demnach darin, daß sie „als Regierungsorganisation außerhalb der Hauptlinien der Abteilungs- und Ministerialhierarchien fällt" und daß sie "daher einen gewissen Grad an Quasiautonomie in ihren täglichen Aktivitäten haben" (Loxley und Saul 1975, 55). Historisch betrachtet verdankt das Parastatal sein Entstehen der Notwendigkeit, ausländisches Kapital und ausländische Expertise in das soeben unabhängig gewordene Entwicklungsland zu ziehen, in dem Staatsmacht und Wirtschaftsmacht von unterschiedlicher Nationalität sind. So meinte rückblickend im Jahr 1974 der Vorsitzende der "Nationalen Bank of Commerce“ in Tansania, A. S. Nsekela: „Beinahe alle unsere Regierungen reagierten anfangs (auf die Situation der wirtschaftlichen Ohnmacht) mit einer Serie von Maßnahmen, die mehr Auslandskapital anziehen sollten, und eine davon war die Gründung oder die Förderung der öffentlichen Unternehmung " (Nsekela 1974, 110/111).

Seither ist wohl in allen afrikanischen Staaten diese institutionalisierte Form einer transnationalen Herrschaftsallianz gediehen. Um die Bedeutung dieses von ihr geprägten Wirtschaftssektors zu zeigen, wird hier aus einem staatlichen „Report on Public Services and the Parastatal Sector" einer früheren Bergbau-Kolonie zitiert: „Aus 
der vorangegangenen Analyse ist wohl ersichtlich geworden, daß der halbstaatliche Sektor in Sambia zu einem komplexen und schwerfälligen Riesen angewachsen ist. Seit den ersten Serien von (staatlichen) Wirtschaftsreformen im Jahr 1968 hat er die sambische Wirtschaft beherrscht und trägt jährlich sicherlich gut über die Hälfte zum BSP bei. Im Jahr 1968 hatte INDECO (Industrial Development Corporation) einen gesamten Umsatz von weniger als 2 Mio. Kwacha ( $1 \mathrm{~K}$ wacha $=3-4 \mathrm{DM})$, um 1973 war er auf 286 Mio. Kwacha angewachsen, und der Umsatz des ZIMCOKomplexes (Zambia Industrial and Mining Corporation) ist jetzt in der Gegend von 1000 Mio. Kwacha im Jahr. Der gesamte Anteil der ,Parastatals' an der LohnBeschäftigung beträgt wenigstens ein Drittel der nationalen Gesamtzahl, und im September 1972 ergab eine Untersuchung des ,National Institute of Public Administration', daß es 292 sambische Graduierte in den privaten und ,parastatal' Sektoren gab (vor allem in letzterem), im Vergleich zu 333, die im öffentlichen Dienst arbeiteten. Aller Wahrscheinlichkeit nach ist jetzt der öffentliche Sektor schon ein ,Minderheits-Aktionär ${ }^{`}$ in der Nutzung von hoch-qualifiziertem einheimischen Personal" (Report 1975, 134).

Selbst in Tansania - dem Staat Schwarzafrikas, der privaten Auslandsinvestitionen reserviert gegenübersteht - ist der „parastatal“ Wirtschaftssektor von großer Bedeu-* tung. Im Jahr 1972 waren in ihm 80000 Lohnarbeiter beschäftigt oder ca. 20\% aller permanent Beschäftigten; $25 \%$ aller Löhne wurden hier bezahlt. Ferner beherrscht der halbstaatliche Sektor auch in Tansania „die mehr dynamischen Sektoren der Wirtschaft" und "steuert 45 bis $50 \%$ der gesamten nationalen Bildung von Investitionskapital bei“ (Loxley und Saul, 1975, 57).

Alle uns vorliegenden empirischen Untersuchungen - über die Verhältnisse in Sambia, Tansania, Kenia, Ghana und im Sudan - stimmen darin überein, daß es bisher keiner Staatsbürokratie gelungen ist, das ausländische Management im halbstaatlichen Sektor zu kontrollieren, ihm Investitions- und Planungsrichtlinien vorzuschreiben oder es gar für sozialistische Ziele funktional einzusetzen ${ }^{7}$. Der tansanische Bankvorsitzende Nsekela resümiert, „daß das öffentliche Unternehmen in Afrika oftmals nicht als ein Mittel zum Aufbau der Ökonomie, wie ursprünglich vorgesehen, gedient hat, sondern als ein viel effizienteres Instrument der Ausbeutung und Unterentwicklung; wirksamer als das rein private Unternehmen, das sich selbst überlassen ist . . . Denn die reale Macht liegt nicht in den öffentlichen Unternehmungen, sondern bei dem ausländischen Management und den Aktionären der assoziierten Unternehmen, deren Führungsstile und persönliche Lebensstile einige unserer Landsleute bedauerlicherweise so begierig nachzuahmen bestrebt sind" $(1974,112)$.

Der halbstaatliche Sektor übt offenbar auf die Beamten und Technokraten des staatlich-administrativen Sektors eine Sogwirkung aus, die bewußtseinsmäßig mit einem Korrumpierungsprozeß Hand in Hand geht. So hat Philip C. Packard in Tansania beobachtet, „daß der halbstaatliche Sektor von vielen Staatsbeamten wegen seiner zusätzlichen Sozialvergütungen bevorzugt wird . . . Darüber hinaus zeigen die Angestellten in ihrer Rolle (als ,parastatal Officer') einen Sinn für Klasse und Privilegien ... Es ist vielleicht $\mathrm{zu}$ früh, davon $\mathrm{zu}$ sprechen, daß sich die ,Parastatals“ als verbriefte Interessen (,vested interests ${ }^{c}$ ) herausbilden, aber Tendenzen dazu gibt

\footnotetext{
7 Uberhaupt ist die Effektivität der legislativen Gewalt in Afrika als gering einzuschätzen. So kommt B. O. Bryde, der diese Aspekte im gegenwärtigen Afrika aus juristischer Sicht untersucht hat, zu dem Schluß: „It is not a lack of legal tools or concepts, but rather the social power structure that limits the role of law in development ... While the political structure of African societies may enable the ruling group to legislate according to their whim, even against the interest of powerful groups, the chances to enforce such rules are small“ (1976, S. 191-192).
} 
es schon, und sie sind sozialistischen Zielsetzungen konträr entgegengesetzt." (Packard 1974, 80).

Ganz ähnliche Tendenzen sind in Kenia und Sambia festgestellt worden. In Sambia versucht man jetzt, dem ,brain drain' von der Staatsbürokratie zum ,parastatal' Sektor durch eine Angleichung der Gehälter entgegenzuwirken. Der Unterschied bei den Spitzengehältern beträgt $1000 \mathrm{Kwacha}$ im Jahr, das ist die Differenz zwischen 10000 K für Parastatal Officers und 9000 K Höchstgehalt im Jahr für Civil Servants (Report 1975, 124). Derselbe Untersuchungsbericht kritisierte, „daß die Regierung mit offensichtlichem Enthusiasmus fortgefahren hat, halbstaatliche Organisationen zu gründen und sie mit erstaunlichen Machtbefugnissen auszustaffieren, während sie gleichzeitig ihre Leistung beklagt und für mehr Kontrollen plädiert" (S. 135). Ein Zeugnis staatlicher Pseudo-Autonomie!

Hier stellt sich die interessante Frage, wie sich das Verhältnis zwischen dem domiranten halbstaatlichen Wirtschaftssektor und den privaten einheimischen Sektoren weiterentwickeln wird. Folgende Hypothese gilt es zu prüfen: einerseits fungiert der halbstaatliche Sektor als Reproduktionsbasis jener partizipierenden, wenig produktiven Staatsbourgeoisie mit stark ausgeprägter Kompradoren-Funktion, andererseits aber möglicherweise auch als Lern- und Rekrutierungsfeld für ein einheimisches Unternehmertum. Dieses trägt - sofern es überhaupt existenzfähig ist - folgende Funktions- und Klassenmerkmale: es gedeiht nur im politischen Schutz der bürokratischen Staatsbourgeoisie und nimmt in den ihr übriggelassenen Sektoren monopolistische Züge an, ferner ist sie in technischer und konjunktureller Hinsicht von der internationalen Bourgeoisie stark abhängig. Gegen diese ist sie auf absehbare Zeit nicht konkurrenzfähig und gedeiht vor allem in deren Kielwasser als KlientelBourgeoisie (wenigstens solange sie von Weltmarktrezessionen verschont bleibt). Colin Leys hat diese Klasse die "monopolistic petty bourgeoisie“ genannt und sie als Produkt des begrenzten „afrikanischen Kapitalismus“ analysiert.

Andere Autoren vertreten die Meinung, daß die gegenwärtige Interessenallianz zwischen Staatsbürokratie, lokaler und internationaler Bourgeoisie in Afrika hauptsächlich widersprüchlichen und daher instabilen Charakter hat. So akzeptiert heute z. B. der nigerianische Staat ausländische Privatinvestitionen nicht mehr wie früher ohne Beschränkungen, sondern nur noch in der Erwartung "as partners in progress led by the public sector" (Akeredolu-Ake, 1975, 27). Dabei ist die Erstarkung des halbstaatlichen Sektors keineswegs als Selbstzweck gedacht, etwa als Vorstufe einer „nicht-kapitalistischen“ Entwicklung, sondern als Voraussetzung für die Schaffung einer einheimischen Industriebourgeoisie (zunächst in den weniger dynamischen Sektoren). Doch auch hier stellt sich wieder die Frage: hätte eine staatlich hochgepäppelte einheimische Unternehmerklasse dauerhaft die Chance und die Kraft, gegen die Konkurrenz des Auslandskapitals sich zu behaupten - was einem ökonomischen Vatermord gleichkäme -, und wenn ja, unter welchen Bedingungen?

Anhand des nigerianischen Beispiels hat Akeredolu-Ake beschrieben, wie die lokale Staatsbürokratie mit Hilfe des Auslandskapitals ein einheimisches gleichrangiges Unternehmertum heranzuzüchten bestrebt war. Dieser zunächst erfolgreiche Prozeß tendierte aber „in späteren Etappen“ dazu, „konkurrierenden und antagonistischen“ Charakter anzunehmen: „je expansiver das private Auslandskapital in diesen späteren Etappen wird, desto weniger Raum ist da für die Entwicklung eines einheimischen Unternehmertums“. Und je schärfer der "Antagonismus" zwischen der internationalen Bourgeoisie im „large-scale“ Wirtschaftssektor und der einheimischen Bourgeoisie 
auf dem Niveau der „small scale“-Industrien wird, desto „tückischer macht sich das Gesetz der komparativen Vorteile bemerkbar (über die anwachsende Kluft in den Kapazitäten zwischen den Sektoren, die zunehmende Spezialisierung und die bloße Trägheit auf seiten der einheimischen small-scale-Geschäftsleute)“ (Akeredolu-Ake 1975, 101-102).

Auch die Beobachtungen in anderen EL mit Tendenzen zur abhängigen Industrialisierung lassen Zweifel als berechtigt erscheinen, ob auf solche Weise - durch Versuche eines protektionistischen, produktions- und wettbewerbsregulierenden Staatsinterventionismus im Interesse staatsnaher einheimischer Bourgeoisiefraktionen - eine nationale Industriebourgeoisie in Afrika entstehen kann, die ihre Existenz nicht primär ihrer abhängigen untergeordneten Weltmarktfunktion verdanken würde. Die heute auch in Afrika sich durchsetzenden Entwicklungsstrategien - bei denen die Weltmarktintegration zur strategischen Größe für Entwicklung wird - lassen die oben angeführte These von M. von Freyhold als zutreffend erscheinen: die Staatsbürokratie und die von ihr bevorzugten einheimischen Bourgeoisiefraktionen können ihrer Unterordnung unter die metropolitane Bourgeoisie mit ihrem überlegenen Produktions- und Marktpotential nicht entrinnen ${ }^{8}$.

Schließlich ist noch die Frage zu prüfen, wieweit die hier abgeleiteten Produktionsund Klassenverhältnisse Möglichkeiten zur strukturellen Veränderung in sich bergen. Obwohl diese Frage hier nicht annähernd erschöpfend behandelt werden kann, scheint es gerechtfertigt zu sein, vor allem Funktion und politische Orientierung des Bergbau- und Industrieproletariats zu betrachten (an zweiter und dritter Stelle müßten wohl Studenten und Kulaken-Bauern untersucht werden); denn die permanent beschäftigten Lohnarbeiter stellen trotz ihrer zahlenmäßigen Schwäche (10 bis 20\% der Erwerbsbevölkerung) die konfliktfähigste Oppositionsgruppe im dominanten Wirtschaftssektor dar. Diese Klasse bezeichnen die einen als konsumorientierte "Arbeiteraristokratie“ (Arrighi und Saul und zahlreiche Nachfolger), die anderen als „populistische Militante“ (A. Peace), als „Reference groups“, die auch von anderen Unterschichten unterstützt wird, die eine Anti-status-quo-Ideologie haben.

Bemerkenswerterweise hat einer der Urheber der These von der afrikanischen „Arbeiteraristokratie“ (die besagt, daß die "skilled“ und "semi-skilled“ Schichten der Arbeiterklasse zusammen mit den Bürokraten zu den privilegierten einheimischen Klassen zählen) kürzlich selbst diese These relativiert, indem er auf die explosive Dialektik der heutigen Industrialisierungsvorgänge in Afrika hinwies: „Die Möglichkeit des neo-kolonialen Wirtschaftssystems, Erfolge hervorzubringen, ist im gegenwärtigen Afrika eng begrenzt; angesichts des Ausbleibens von Strukturreformen die sozialistischen Strategien vorausgehen - , sind Krisen und/oder Stagnation unvermeidlich, und, damit verbunden, erweist sich die Kooptation selbst der stabilsten Sektionen der Arbeiterklasse als sehr viel schwieriger (als in den 60er Jahren).

\footnotetext{
8 In seiner empirischen Untersuchung der Entwicklungsprozesse in vier afrikanischen Ländern kommt H. Neitzel zu dem Ergebnis: „Die Industrialisierungspolitik der Regierungen dieser beiden Staaten (Senegal und Elfenbeinküste) konnte sich deshalb im großen und ganzen auf die Schaffung weiterer Investitionsanreize wie Steuerbefreiungen, Zollsenkungen für Maschinen- und Rohstoffimporte sowie die Bereitstellung zinsgünstiger Kredite beschränken ... In der Realität waren diese Maßnahmen allerdings ziemlich bedeutungslos, da alle westafrikanischen Staaten solche Investitionsanreize geschaffen hatten ... Dagegen hat sich die Integration der Industrie in die Weltwirtschaft verstärkt, und die Abhängigkeit vom Ausland ist gewachsen. Dafür ist in erster Linie der hohe Anteil ausländischer Privatinvestitionen verantwortlich, deren Problematik und Konsequenzen diskutiert wurden. Daneben ist als eine Folge der Industrialisierung der Bedarf an Investitionsgütern, Halbfertigwaren und Energiestoffen stark gestiegen, die importiert werden müssen. Dementsprechend wirkt ein erheblicher Teil der von der Industrie ausgelösten Nachfrageimpulse nicht innerhalb des Landes, sondern im Ausland - meist in Westeuropa. Die an die Industrialisierung geknüpften Erwartungen, über direkte und indirekte Effekte einen wesentlichen Beitrag zur Entwicklung der Wirtschaftsstruktur zu leisten, haben sich nicht erfüllt." (Neitzel 1976, 86-89.)
} 
Unter solchen Umständen wird das Ausmaß an falschem Bewußtsein weniger dramatisch sein und einige der klassischen Stärken der städtischen Arbeiterklasse eher sichtbar . . . Dann wird sich auch die obere Schicht der Arbeiter höchstwahrscheinlich ,nach unten' identifizieren und so eine führende Kraft innerhalb der revolutionären Allianz der ausgebeuteten Elemente in der Gesellschaft werden "! (J. Saul 1975, 306).

In die gleiche Richtung weisen neuere Untersuchungen über die Arbeiterproteste in Afrika, die ergaben, daß Generalstreiks und städtische Revolten im Sudan, in Äthiopien, in Kongo-Brazzaville, in Ghana und Nigeria Ziele verfolgten, die über begrenzte wirtschaftliche Zugeständnisse hinausgingen (M. Ottaway 1976). Sandbrook und Cohen unterstreichen dabei die Tatsache, daß „alle diese Manifestationen von Arbeiterprotesten politische Krisen erzeugten oder zu ihnen beitrugen; sie wirkten als Flammpunkt von beginnender, aber weit verbreiteter Unzufriedenheit mit den Realitäten der politischen Unabhängigkeit" (1975, 201). Auch die Beobachtungen von Adrian Peace in Nigeria - einem Land mit reicher proletarischer Erfahrung sind besonders aufschlußreich. Er charakterisiert das Proletariat in Lagos als „örtlich verankerte politische Elite der städtischen Massen“, „als eine ,reference group“ in politischen Angelegenheiten für andere städtische Schichten, die . . . von der Lohnarbeiterklasse die Artikulation von politischem Protest gegen eine in höchstem Maße ungleiche Gesellschaft erwarten“ (1975, 289).

Gerade ein Vergleich der gespannten Beziehungen zwischen Staatsbourgeoisie und Industrieproletariat im neokolonialen Nigeria einerseits und in Tansania mit seiner „sozialistischen“ Entwicklungsdoktrin andererseits läßt die Schlußfolgerung zu, daß sich die Staatsbürokratie beider Ländertypen in dem oben beschriebenen prekären Belagerungszustand befindet: einerseits wird sie immer stärker von den frustrierten nationalen Unterschichten belagert und bedroht, angeführt von radikalisierten Studenten und einem klassenbewußter werdenden städtischen Industrieproletariat, und andererseits hat sie der metropolitanen Bourgeoisie (den multinationalen Konzernen) ein Kooperations- und Investitionsklima zu gewährleisten, das die Expansion des Wirtschaftswachstums sowie den gesicherten Gewinntransfer erlaubt (siehe Neitzel 1976). Was die Situation für die Staatsbourgeoisie noch schwieriger macht, ist die Tatsache, daß auf der Oberfläche der sozialen Beziehungen das Auslandskapital mit seinen Investitionen in den Wachstumsbranchen (aufgrund höherer Lohnzahlungen und vergleichsweise großen "fringe benefits") als relativ "progressiv" erscheint. Dies macht es der bürokratischen Staatsbourgeoisie wiederum noch schwerer, die materielle, berufliche und soziale Misere der Massen mit ausländischen „neo-kolonialistischen Machenschaften“ zu begründen. So weitet sich noch der politische „credibility gap“ zwischen einer ohnmächtigen und meist korrupten Staatsbourgeoisie und den frustrierten, vom Staat unterdrückten oder benachteiligten Klassen und Produktionsgemeinschaften - eine krisenträchtige Situation, die reif ist für die Machtübernahme des Militärs.

\section{Ausblick}

Zugegebenermaßen stimmen die hier vorgebrachten Überlegungen über die Entwicklungsperspektiven in den afrikanischen Staaten südlich der Sahara alles andere als optimistisch. Beim heutigen Stand der "Entwicklung“ jedoch, die durch verstärkte Weltmarktabhängigkeit und Außenorientierung der Produktionsverhältnisse (Senghaas 1977) sowie durch zunehmende staatsbürokratische Intervention und Repression 
gekennzeichnet ist, gibt es wenig Grund, die afrikanische "Staatsklasse“ - so unterschiedlich im einzelnen ihr politischer Charakter sein mag — als geeigneten „Agenten für Entwicklung“ anzusehen. Zwar, umfaßt ihre Funktion im Reproduktionsprozeß sowohl wachstumsfördernde als auch parasitäre und kompradorenhafte Elemente, aber ihre "produktive" Tätigkeit konzentriert sich mit zunehmender Aktivität auf das, was als Revenue-Beschaffungsfunktion abgeleitet wurde. Dabei liegen die Ursachen für diese reformunfähige Politik vor allem noch in der historischen Schwäche der regierenden Klasse als überwiegend bürokratische und partizipierende Bourgeoisie (ohne Kontrolle über die Produktionsbedingungen). Dieser Geburtsfehler macht sie noch immer materiell und politisch - aufgrund spezifischer Revenuequellen abhängig von den kolonialen/neokolonialen Produktions- und Klassenverhältnissen und somit auch von der metropolitanen Bourgeoisie.

In dem durchaus feststellbaren Bestreben der Staatsklasse, wirtschaftliches Wachstum, Infrastrukturinvestitionen und den Konsum zu intensivieren, setzt sie im Prinzip den Weg der sektorell ungleichen Kapitalisierung von Ökonomie und Gesellschaft fort, den ihre kolonialen Vorgänger mit der metropolitan bedingten Zersetzung vorkapitalistischer Produktionsverhältnisse begonnen haben. Das somit höchstens modifizierte Muster abhängiger Entwicklung (Importsubstitutions-Industrialisierung, Exportdiversifizierung) ist daher ungeeignet, um a) der lokalen Staatsklasse die notwendige Kontrollgewalt über die nationalen Produktionsprozesse zu bringen als Voraussetzung für eine Politik der nationalen "Self-Reliance“ —, und um b) sich als herrschende Klasse gegenüber den Erwartungen rivalisierender nationaler Bourgeoisiefraktionen und den diversen Produktionsgemeinschaften in den vernachlässigten Wirtschaftssektoren dauerhaft zu legitimieren.

Im krisenträchtigen Zustand zweiseitiger Belagerung rettet die Staatsklasse buchstäblich ihre Haut durch a) intensivierte "Entwicklungskooperation“ mit dem Ausland, oftmals zu beschämenden Bedingungen und für den Preis der Stabilisierung von national keineswegs entwicklungskonformen Produktionsverhältnissen, und b) durch zunehmende Repression nach innen, mit der Folge wachsender Spannungen zwischen Staatsbürokratie und ihren "supportive classes“ einerseits und den deprivierten staatsfernen Klassen (Industrieproletariat) und Sektoren (Landwirtschaft) andererseits. Dabei vollzieht sich diese Entwicklungskooperation zwischen lokaler Staatsklasse und metropolitaner Bourgeoisie - obwohl sie je nach der Ressourcenstärke des EL mit unterschiedlichen Nutzeffekten zustande kommt - in der Regel nach einem Grundtausch etwa folgenden Musters: Entwicklungskredite, Budgethilfe, Produktrenten und Gewinnbeteiligung gegen Rohstoffe, Marktrechte und gesichertes Investitionsklima.

Die Herrschaftsformen, in denen sich die Staatstätigkeit manifestiert, sind zum einen die staatsbourgeoise Partizipation an der Produktion der Auslandsunternehmen, die im dominanten "halbstaatlichen“ Wirtschaftssektor die Kontrollfunktion behalten, und zum anderen die staatliche Allokationspolitik, die die knappen Sektorressourcen und Staatsrevenuen eher nach politischen denn nach entwicklungskonformen Kriterien einsetzt.

Soweit die oben dargestellten Zusammenhänge zwischen Staatsfunktionen und Klassenstrukturen die gegenwärtigen Entwicklungstendenzen in afrikanischen Ländern wenigstens tendenziell richtig erfassen (von Ländern wie Mozambique, Guinea- Bissau, Angola, aber auch Uganda abgesehen), müßten u. E. weitere Einzelanalysen 
folgende Hypothesen berücksichtigen, die mehr als Denkanstöße denn als gesicherte und überall zutreffende Aussagen aufzufassen sind:

1. Der periphere Staat bzw. die bürokratische "Staatsklasse“ in Afrika ist gegenwärtig nicht nur wenig geeignet, als „Agent für Entwicklung“ zu fungieren, sondern stellt eher das interne Haupthindernis für eine Entwicklung dar, die national vorhandene Ressourcen und Produktivkräfte im Dienste einer gesamtgesellschaftlichen Bedürfnisbefriedigung zu entwickeln hätte (vgl. I. L. Markovitz 1976).

2. Die "herrschende Klasse“ in Afrika ist je nach Typ des betreffenden EL ein transnationales, wenn auch keineswegs konfliktfreies und stabiles Interessebündnis, das aus a) der lokalen Staatsbürokratie, b) diversen Repräsentanten der metropolitanen Bourgeoisie und c) rivalisierenden lokalen Bourgeoisiefraktionen und Produzentengemeinschaften unterschiedlicher Wirtschaftssektoren besteht.

3. Der periphere Staat eines EL ist nicht nur als lokal begrenzter Herrschafts-, Interventions- und Verteilungsapparat $\mathrm{zu}$ begreifen, sondern vor allem in seiner funktionalen und herrschaftssoziologischen Einbettung in ein internationales hierarchisch strukturiertes Dominanz- und Dependenzsystem. Aufgrund der Präsenz der internationalen Organisationen mit staatlichen Ersatz- und Ergänzungsfunktionen in den Peripherien (wie IWF, Weltbank, multinationale Konzerne etc.) ergibt sich die Wirksamkeit eines strukturell heterogenen "Staates“, von dem der periphere institutionell erfaßbare Staat nur ein integraler Bestandteil ist.

4. Mit zunehmender Internationalisierung der Staatsfunktionen in den Peripherien entwickeln sich Formen eines weltmarktassoziierten "Staatskapitalismus“, der trotz aller induzierter Wirtschaftsdynamik mehr soziale Spannungen hervorruft, als er zu lösen vermag. Demzufolge werden militantere Formen des klassenbewußten Protestes von seiten der Arbeiterorganisationen, von Studenten und anderen absolut oder relativ deprivierten Klassen und sektoralen Produktionsgemeinschaften (Bauern) eher zu- als abnehmen. Die „Lateinamerikanisierung“ der afrikanischen Gesellschaftsverhältnisse im Sinne zunehmender sozialer Polarisierung und nationaler Desintegration steht zu befürchten (Tetzlaff 1976).

5. Aufgrund ihrer historisch bedingten sozio-ökonomischen Schwäche, ihrer permanenten Revenueknappheit sowiq der daraus resultierenden asymmetrischen Koalition mit der metropolitanen Bourgeoisie ist die bürokratische Staatsklasse in den Ländern Schwarzafrikas objektiv nicht in der Lage, die Produktionsbedingungen im eigenen Lande in Hinblick auf eine Revision der deformierten Strukturen umzuformen. Es ist vielmehr anzunehmen, daß der eingeschlagene Weg der „Entwicklung“ via Weltmarktintegration für eine absehbare Zeit fortgesetzt werden wird. Die historischen Realisierungschancen für Strategien autozentrierter Entwicklung scheinen daher äußerst gering. Trotz zunehmender interner Turbulenzen ist keine soziale Gegenmacht in Sicht, die die herrschende Klassenkoalition überwinden könnte.

Daher ist der verbleibende Veränderungsspielraum der Staatsklasse in erster Linie extern abhängig von den Freiräumen, die durch den Strukturwandel in den Metropolen entstehen (Auslagerungsindustrien, komplementäre Arbeitsteilung etc.). Das Lomé-Abkommen zwischen der Europäischen Gemeinschaft und den 46 AKP-Staaten von 1975 steckt in etwa den Rahmen ab, in dem sich die heute möglichen Veränderungen in der asymmetrischen Arbeitsteilung zwischen Westeuropa und Afrika bewegen können. 


\section{Literatur}

Albertini, Rudolf v. Dekolonisation. Die Diskussion über die Verwaltung und Zukunft der Kolonien 1919-1960, Köln und Opladen 1966

Ake, Claude Explanatory Notes on the Political Economy of Africa, in: The Journal of Modern African Studies, Vol. 14, Nr. 1 (1976), S. 1-23

Akeredolu-Ale, E. O. The Underdevelopment of Indigenous Entrepreneurship in Nigeria, Ibadan 1975

Alawi, Hamza The State in Postcolonial Societies: Pakistan and Bangladesh, in: K. Gough and H. P. Shauna (ed.), Imperialism and Revolution in South Asia, New York 1973, S. 145-173

Amin, Samir Die ungleiche Entwicklung. Essay über die Gesellschaftsformationen des peripheren Kapitalismus, Hamburg 1975

Arrighi, Giovanni and Saul, John S. Essays on the Political Economy of Africa, New York and London 1973

Baer, W. et. al. The Changing Role of the State in the Brazilian Economy, in: World Development, Oxford, Nr. 11 (Nov. 1973), S. 23-34

Barnet, Richard J. u. Müller, Ronald E. Die Krisenmacher. Die Multinationalen und die Verwandlung des Kapitalismus, Reinbek bei Hamburg 1975

Basso, Lelio Gesellschaftsformation und Staatsform. Drei Aufsätze, Ffm 1975

Blanke, B., Jürgens, U. u. Kastendiek, H. Zur neueren marxistischen Diskussion über die Analyse von Form und Funktion des bürgerlichen Staates. Überlegungen zum Verhältnis von Politik und Ökonomie, in: Probleme des Klassenkampfes 14/15, Jg. 1974, Nr. 3, S. 51-104

Braunmühl, Funken, Cogoy, Hirsch Probleme einer materialistischen Staatstheorie, Ffm 1973

Bryde, Brun-Otto The Politics and Sociology of African Legal Development, Ffm 1976

Cabral, Amilcar Die Revolution der Verdammten, Berlin 1974

Daaku, Kwame Yeboa Trade and Politics on the Gold Coast 1600 to 1720. A. Study of the African Reaction to European Trade, Oxford 1970

Diop, Majhemout Classes and Class Ideology in Senegal, Dakar 1966

Dumont, René False Start in Africa, London 1967 (Paris 1962)

Elsenhans, Hartmut Zur Rolle der Staatsklasse bei der Überwindung von Unterentwicklung, in: A. Schmidt, a. a. O., S. 250-265

Evers, Tilman Unterentwicklung und Staat. Elemente einer Theorie des Staates im peripheren Kapitalismus, Diskussionspapier, vorgelegt zum Kolloquium über Urbanisierungsprobleme im Okt. 1975 in Gießen. FB Gesellschaftswissenschaften

Fanon, Franz Die Verdammten dieser Erde, Ffm 1966

Farsoun, Samih and Carroll, Walter Der Bürgerkrieg im Libanon. Sekten, Klassen u. Imperialismus, in: Monthly Review, 2. Jg., H. 2 (Juli/August 1976), dt. Ausgabe, S. 12-37

Feldman, Rayah Rural social differentiation and political goals in Tanzania, in: I. Oxaal, T. Barnett, D. Booth (ed.), Beyond the sociology of development. Economy and society in Latin America and Africa. London und Boston 1975, S. $154-182$ 
First, Ruth Power in Africa, New York 1970

First, Ruth The Barrel of a Gun. Political Power in Africa and the Coup d' Etat, Harmondsworth 1972

Freyhold, Michaela v. The Post-colonial State and its Tanzanian Version. Contribution to a Debate, Manuskript, Dar es Salaam 1975

Gerstenberger, Heidi Klassenantagonismus, Konkurrenz und Staatsfunktionen, in: Gesellschaft. Beiträge zur Marx'schen Theorie 3, Ffm 1975, S. 7-26

Good, Kenneth Settler Colonialism: Economic Development and Class Formation, in: The Journal of Modern African Studies, Vol. 14, Nr. 4 (1976), S. $597-620$

Green, Reginald H. und Seidmann, Ann Unity or Powerty? The Economics of Pan-Africanism, Harmondsworth 1968

Grewe, Hartmut Ein austauschtheoretisches Paradigma zur Analyse international verflochtener Politikbereiche von Entwicklungsländern, Diskussionspapier für den AK „Entwicklungspolitik und Entwicklungstheorie“, Nov. 1974

Grohs, Gerhard Stufen afrikanischer Emanzipation. Studien zum Selbstverständnis westafrikanischer Eliten, Stuttgart etc. 1967

Grohs, Gerhard und Tibi, Bassam (Hrsg.) Zur Soziologie der Dekolonisation Afrikas, Ffm 1973

Guggenberger, Bernd Wem nützt der Staat? Kritik der neomarxistischen Staatstheorie, Stuttgart etc. 1974

Harris, Richard (ed.) The Political Economy of Africa, New York etc. 1975

Hein, Wolfgang u. Simonis, Georg Entwicklungspolitik, Staatsfunktionen und Klassenauseinandersetzungen im peripheren Kapitalismus, in: A. Schmidt, a. a. O., S. $216-249$

Hermann, Klaus Gesellschaftsklassen und Gesellschaftsschichten im tropischen Afrika, in: K. Meschkat und O. Negt (Hrsg.), Gesellschaftsstrukturen, Ffm 1973, S. 294-319

Hill, Polly Studies in Rural Capitalism in West Africa, Cambridge 1970

ILO (Hrsg.) Employment, incomes and equality. A Strategy for increasing productive Employment in Kenya, Genf 1972/1974

Kilson, Martin British Colonialism and Transformation of Traditional Elites: Case of Sierra Leone, in: W. Cartey and M. Kilson (ed.), The African Reader: Colonial Africa, New York 1970

Kitching, Gavin N. The Concept of Class and The Study of Africa, in: The African Review, Vol. 2, Nr. 3, 1972, S. 327-350

Kuper, Leo Race, Class and Power: Ideology and Revolutionary Change in Plural Societies, London 1974

Ledda, Romano Social Classes and Political Struggle in Africa, in: International Socialist Journal, August 1967

Lele, Uma The Design of Rural Development. Lessons from Africa, Baltimore and London 1975

Leys, Colin The Limits of African Capitalism, The formation of the monopolistic petty bourgeoisie in Kenya (mimeographed), Nairobi, 1972

Leys, Colin Underdevelopment in Kenya: The Political Economy of Neo-colonialism, London 1975

Lloyd, Peter C. The New Elites of Tropical African, London 1966/1970

Lloyd, Peter C. Classes, Crises and Coups, London 1971 
Loxley, John und Saul, John S. Multinationals, Workers and Parastatals in Tanzania, in: Review of African Political Economy, Nr. 2 (Jan.-April 1975), S. $54-88$

Mamalakis, Markos The Theory of Sectoral Clashes and Coalitions Revisited, in: Latin American Research Review, Nr. 6 (1971), S. 89-126

Manghezi, Alpheus Class, Elite and Community in African Development, Scandinavian Institute of African Studies, Uppsala 1976

Markovitz, Irving L. Bureaucratic Development and Economic Growth, in: The Journal of Modern African Studies, Vol. 14, Nr. 2 (1976), S. 183-200

McGowan, Patrick J. Economic Dependence and Economic Performance in Black Africa, in: The Journal of Modern African Studies, Vol. 14, Nr. 1 (1976), S. $25-40$

Miller, Robert A. Elite Formation in Africa: Class, Culture, and Coherence, in: The Journal of Modern African Studies, Vol. 12, Nr. 4 (1974), S. 521-542

Murray, Robin Der Nationalstaat und die Internationalisierung des Kapitals, in: O. Kreye (Hrsg.), Multinationale Konzerne. Entwicklungstendenzen im kapitalistischen System, München 1974, S. 40-80

Myrdal, Gunnar Politisches Manifest über die Armut in der Welt (Kap. 7: „Der schwache Staat"), Ffm 1970

N’Dongo, Sally „Coopération“ et néo-colonialisme, Paris 1976

Neitzel, Hartmut Industrialisierung als Entwicklungsstrategie in Westafrika. Dargestellt am Beispiel der Länder Elfenbeinküste, Mali, Obervolta und Senegal, Arbeiten aus dem Institut für Afrikakunde, Heft 8, Hamburg 1976

Nkrumah, Kwame Nsekela Class Struggle in Africa, London 1970

Ocampo, José u. Johnson, Dale L. The Concept of Political Development, in: James D. Cockcroft u. a., Dependence and Underdevelopment: Latin America's Political Economy, Garden City, N. Y. 1972, S. 399-424

Okwuosa, Emanuel New Direction for Economic Development in Africa, Surrey 1967

Ottaway, Marina Social Classes and Corporate Interests in the Ethopian Revolution, in: The Journal of Modern African Studies, Vol. 14, Nr. 3 (Sept. 1976), S. $469-486$

Packard, Philip C. Management and Control of Parastatal Organisations, in: Towards Socialist Planning, Tanzanian Studies No. 1, Dar es Salaam 1974, S. 73-91

Peace, Adrian The Lagos Proletariat: Labour Aristocrats or Populist Militants, in: R. Sanbrook and R. Cohen, a. a. O., S. 281-302

Report of the Commission of Inquiry into the Salaries, Salary Structures and Conditions of Service, Vol. I, The Public Services and the Parastatal Sector, hrsg. v. Republic of Zambia, Lusaka 1975

Rodney, Walter How Europe underdeveloped Africa, Dar es Salaam und London 1972

Rweyemamu, Justinian Underdevelopment and Industrialization in Tanzania. A Study of Perverse Capitalist Industrial Development, Nairobi etc. 1973

Sandbrook, Richard and Cohen, Robin (ed.) The Development of an African Working Class. Studies in Class Formation and Action, London 1975

Saul, John S. The "Labour Aristocracy" Thesis Reconsidered, in: R. Sanbrook and R. Cohen, a. a. O., S. 303-310 
Schmidt, Alfred (Hrsg.) Strategien gegen Unterentwicklung. Zwischen Weltmarkt und Eigenständigkeit, $\mathrm{Ffm} / \mathrm{New}$ York 1976

Senghaas, Dieter (Hrsg.) Peripherer Kapitalismus. Analysen über Abhängigkeit und Unterentwicklung, Ffm 1974

Senghaas, Dieter Weltwirtschaftsordnung und Entwicklungspolitik. Plädoyer für Dissoziation, Ffm 1977

Shivji, Issa G. The Silent Class Struggle, Class Struggles in Tanzania, Dar es Salaam 1975, Tanzanian Studies No. 2, Dar es Salaam 1974

Sonntag, Heinz Rudolf Der Staat des unterentwickelten Kapitalismus, in: Kursbuch Nr. 31 (Mai 1973), S. 157-183

Stavenhagen, Rodolfo Social Classes in Agrarian Societies, New York 1975

Szentes, Tamás Politische Ökonomie der Entwicklungsländer, Ffm u. Köln 1974

Tetzlaff, Rainer Multinationale Konzerne und politische Systeme in Entwicklungsländern: die "Lateinamerikanisierung“ der Klassenstrukturen in der Dritten Welt, in: D. Senghaas und U. Menzel (Hrsg.), Multinationale Konzerne und Dritte Welt, Opladen 1976, S. 145-169

Van Velzen, T. U. E. Thoden Staff, Kulaks and Peasants: A Study of a Political Field, in: Socialism in Tanzania, Vol. 2 Policies, ed. by L. Cliffe and J. S. Saul, Nairobi 1973, S. 153-79 
try to keep peripheral capitalism going, and governments that come to power by popular movements promoting alternative ways of economic development, to "do something for the underprivileged masses", the article rejects the simplistic notion of the state as a modernizing agent. On the contrary, the concept of bureaucracy as a state class comparable to the state classes of the so-called "asiatic modes of production" should be further elaborated. The central approach is, that a state class does dispose of the mass of economic surplus of society and in opposition to the false notion of administrative bourgeoisie - is not forced by mechanisms of economic competition to invest this surplus in oder to increase productive forces.

The tendencies of formation of a state class with these caracteristics are a necessity in LDC's, because existing markets do not indicate rational choices for investment as income distribution is highly unequal. But the potential benefit of the existence of a state class, that is the possibility of investing funds in order to promote productive forces in the interest of a later increase of mass consumption, is not necessarily realised. The state class can also use the economic surplus for its own conspicuous consumption.

Whether the state class wastes funds or whether it invests funds for mass needs, is not economically determined, but is the outcome of social conflicts influencing the specific mechanisms of the internal rivalries of the state class. The article gives some hints, in which direction these societal dynamics should be further explored.

The article is a further investigation into the political and social dynamics of overcoming underdevelopment the basic economic stratagem of which has been presented in my article on: "Overcoming Underdevelopment. A Research Paradigm," published in the Journal of Peace Research No. 4 Vol. XII/1975, pp. $293-313$.

State and class in peripher-capitalistic societies: the development of dependent state-capitalism in Black Africa

\section{By Rainer TetzlafF}

The article deals with two main scientific issues: how can the function of the state in the development process of LDC be defined, and which are the main classes in peripher-capitalistic societies. Some authors qualify the state in LDC as politically weak, others as relatively strong and independent from social classes. The article stresses the fact that today some important state functions are fulfilled by international organisations; that is why one can formulate the thesis of an internationalisation of state functions in LDC.

In the second part follows a discription of the historical genesis of state functions and class formations in Africa during the period between 1880 and 1960. Five types of colonies can be distinguished, each of them being characterized by specific colonial interests and specific "classes" (migrant workers, cash crop-peasants, mine workers etc.) as the result of colonial rule.

The colonial state had as a main function the dissolution of pre-capitalistic conditions for production. At the eve of independence a bureaucratic and urbanized local petty bourgoisie inherited the colonial state apparatus which 
remained up to the present relatively unchainged, insofar as its functions for the production process were concerned. As a new function, the state has to mobilize revenues in different ways. This increases even more the dependence from metropolitan capitalism. The typical form of the alliance of interests between the local power elite and the international bourgeoisie is the parastatal sector, which is shown by examples from Tanzania and Zambia.

Today we face a silent class struggle between the westernized bureaucratic bourgoisie and its international partners (multinational corporations) on the one hand and industrial workers (Nigeria), kulaks and other social classes on the other hand. The local state in LDC is thereforde in a stage of siege. Hope for changing the present power structures in Black Africa is small.

\section{General Principles of Law in the Constitution of the Buddhist Order (Vinaya)}

\section{By Hellmuth Hecker}

The "Vinaya" (Constitution of the Buddhist Sangha) is one of the most ancient legal documents about the social life of human society. This order emerged 2500 years ago in North India. It's rules which are traced back to Buddha himself have been taken over, with only little accommodations, by all Buddhist schools, as well in South-East-Asia as in Tibet or China. The Vinaya is divided into two parts. The first part contains a wealth of special commandments and permissions. The second part contains 227 numbered rules which form the Penal Code of the order (the so-called Pãtimokkha). The Pãtimokkha is divided into eight categories according to the gravity of the act. Each of the 227 rules is set up according to a uniform scheme which also contains terminological definitions and a collection of cases.

Three fields that show general principles of law have been selected here out of the richness of rules in both parts of the Vinaya:

1. Reasons for exemption from punishment within the Pãtimokkha. Even at that time the principle "nullum crimen sine lege" had been determined explicitly. Furthermore the following subjects are treated: irresponsibility, absence of criminal intent, interruption of attempt, permission, relationship, or parapsychological fact, influence of illness and accidents, as well as other important reasons.

2. Acquisition and forfeiture of membership in the order, which is comparable to the acuisition or forfeiture of nationality. Here are treated: competency, procedure, admission of novices, general conditions of ordination, causes for expatriation (facts of forfeiture).

3. Conflicts and their settlement. Here are illustrated several facts which are considered as a breach of peace. Then follows the description of seven ways of disciplinary measures for the maintenance of order. The procedure of settlement of conflicts is treated in detail. It comprises general settlement principles which are wider than a code of criminal procedure. Finally controversies related to rank in the order are treated.

The English translator of the Vinaya states that in respect of the causes for exclusion of penalty "all are remarkable for their humane and lenient tone, for their reasonableness and common-sense". This may count for all other Vinayaprovisions. 